\title{
Shape of the oxygen abundance profiles in CALIFA face-on spiral galaxies
}

L. Sánchez-Menguiano ${ }^{1,2}$, S. F. Sánchez ${ }^{3}$, I. Pérez ${ }^{2}$, R. García-Benito ${ }^{1}$, B. Husemann ${ }^{4}$, D. Mast ${ }^{5,6}$, A. Mendoza ${ }^{1}$, T. Ruiz-Lara ${ }^{2}$, Y. Ascasibar ${ }^{7,8}$, J. Bland-Hawthorn ${ }^{9}$, O. Cavichia ${ }^{10}$, A. I. Díaz ${ }^{7,8}$, E. Florido ${ }^{2}$, L. Galbany ${ }^{11,12}$, R. M. Gónzalez Delgado ${ }^{1}$, C. Kehrig ${ }^{1}$, R. A. Marino ${ }^{13,14}$, I. Márquez ${ }^{1}$, J. Masegosa ${ }^{1}$, J. Méndez-Abreu ${ }^{15}$, M. Mollá ${ }^{16}$, A. del Olmo ${ }^{1}$, E. Pérez ${ }^{1}$, P. Sánchez-Blázquez ${ }^{7,8}$, V. Stanishev ${ }^{17}$, C. J. Walcher ${ }^{18}$, Á. R. López-Sánchez ${ }^{19,20}$, and the CALIFA collaboration

\footnotetext{
${ }^{1}$ Instituto de Astrofísica de Andalucía (CSIC), Glorieta de la Astronomía s/n, Aptdo. 3004, 18080 Granada, Spain e-mail: 1sanchez@iaa.es

2 Dpto. de Física Teórica y del Cosmos, Universidad de Granada, Facultad de Ciencias (Edificio Mecenas), 18071 Granada, Spain

3 Instituto de Astronomía, Universidad Nacional Autónoma de México, AP 70-264, 04510 México, D.F., Mexico

${ }^{4}$ European Southern Observatory (ESO), Karl-Schwarzschild-Str. 2, 85748 Garching b. München, Germany

5 Instituto de Cosmologia, Relatividade e Astrofísica - ICRA, Centro Brasileiro de Pesquisas Físicas, Rua Dr. Xavier Sigaud 150, CEP 22290-180, Rio de Janeiro, RJ, Brazil

6 Observatorio Astronómico de Córdoba, Universidad Nacional de Córdoba, X5000 BGR Cordoba, Argentina

7 Departamento de Física Teórica, Universidad Autónoma de Madrid, 28049 Cantoblanco, Spain

8 Astro-UAM, UAM, Unidad Asociada CSIC, Faculdad de Ciencias, Campus de Cantoblanco, 28049 Madrid, Spain

9 Sydney Institute for Astronomy, School of Physics A28, University of Sydney, NSW 2006, Australia

${ }_{10}$ Instituto de Física e Química, Universidade Federal de Itajubá, Av. BPS, 1303, 37500-903 Itajubá-MG, Brazil

11 Millennium Institute of Astrophysics MAS, Nuncio Monseñor Sótero Sanz 100, Providencia, 7500011 Santiago, Chile

12 Departamento de Astronomía, Universidad de Chile, Camino El Observatorio 1515, Las Condes, Santiago, Chile

13 CEI Campus Moncloa, UCM-UPM, Departamento de Astrofísica y CC. de la Atmósfera, Facultad de CC. Físicas, Universidad Complutense de Madrid, Avda. Complutense s/n, 28040 Madrid, Spain

14 Department of Physics, Institute for Astronomy, ETH Zürich, 8093 Zürich, Switzerland

15 School of Physics and Astronomy, University of St Andrews, SUPA, North Haugh, KY16 9SS St Andrews, UK

16 CIEMAT, Avda. Complutense 40, 28040 Madrid, Spain

17 Department of Physics, Chemistry and Biology, IFM, Linköping University, 58183 Linköping, Sweden

18 Leibniz-Institut für Astrophysik Potsdam (AIP), An der Sternwarte 16, 14482 Potsdam, Germany

19 Australian Astronomical Observatory, PO Box 915, North Ryde, NSW 1670, Australia

20 Department of Physics and Astronomy, Macquarie University, NSW 2109, Australia
}

Received 25 September 2015 / Accepted 12 December 2015

\section{ABSTRACT}

\begin{abstract}
We measured the gas abundance profiles in a sample of 122 face-on spiral galaxies observed by the CALIFA survey and included all spaxels whose line emission was consistent with star formation. This type of analysis allowed us to improve the statistics with respect to previous studies, and to properly estimate the oxygen distribution across the entire disc to a distance of up to 3-4 disc effective radii $\left(r_{\mathrm{e}}\right)$. We confirm the results obtained from classical $\mathrm{H}$ II region analysis. In addition to the general negative gradient, an outer flattening can be observed in the oxygen abundance radial profile. An inner drop is also found in some cases. There is a common abundance gradient between 0.5 and $2.0 r_{\mathrm{e}}$ of $\alpha_{\mathrm{O} / \mathrm{H}}=-0.075 \mathrm{dex} / r_{\mathrm{e}}$ with a scatter of $\sigma=0.016 \mathrm{dex} / r_{\mathrm{e}}$ when normalising the distances to the disc effective radius. By performing a set of Kolmogorov-Smirnov tests, we determined that this slope is independent of other galaxy properties, such as morphology, absolute magnitude, and the presence or absence of bars. In particular, barred galaxies do not seem to display shallower gradients, as predicted by numerical simulations. Interestingly, we find that most of the galaxies in the sample with reliable oxygen abundance values beyond $\sim 2$ effective radii (57 galaxies) present a flattening of the abundance gradient in these outer regions. This flattening is not associated with any morphological feature, which suggests that it is a common property of disc galaxies. Finally, we detect a drop or truncation of the abundance in the inner regions of 27 galaxies in the sample; this is only visible for the most massive galaxies.
\end{abstract}

Key words. galaxies: abundances - galaxies: evolution - galaxies: ISM - galaxies: spiral - techniques: imaging spectroscopy techniques: spectroscopic

\section{Introduction}

Understanding how disc galaxies form and evolve is one of the greatest challenges in galactic astronomy. Some of the remaining unsolved fundamental questions are comprehending the processes that are involved in the assembly of galaxies of different masses, the relative importance of mergers versus continuous gas accretion infall into the disc, the rate of metal enrichment, and the angular momentum transfers during these processes.

The distribution of gas chemical abundances and stellar parameters as well as their variation in space and time are important tools for answering these questions on the evolution of discs in spiral galaxies. Infall models of galaxy formation predict 
that spiral discs build up through accretion of material, which leads to an inside-out growth (Matteucci \& Francois 1989; Molla et al. 1996; Boissier \& Prantzos 1999). The accretion brings gas into the inner parts of the discs, where it reaches high densities that trigger violent and quite efficient star formation. Thus, there is a fast reprocessing of gas in the inner regions, which leads to a population of old, metal-rich stars surrounded by a high-metallicity gaseous environment, while the outer regions are populated by younger, metal-poor stars formed from poorly enriched material. The first evidence that supports this scenario for disc evolution comes from studies on stellar colour profiles in nearby galaxies, which find bluer colours in the outer parts (e.g. de Jong 1996; MacArthur et al. 2004; Taylor et al. 2005; Muñoz-Mateos et al. 2007). This blueing can be interpreted on the basis of a change in the disc scale-length as a function of the observed wavelength band. This result is predicted by models based on the inside-out growth (Prantzos \& Boissier 2000). To explain the nature of these colour gradients, recent works have analysed the radial change in the star formation history (SFH, Sánchez-Blázquez et al. 2009; Pérez et al. 2013).

Another independent result that is consistent with this scenario comes from the weak dependence of disc galaxies with redshift on the stellar mass-size relation. According to the insideout growth of discs, galaxies are expected to increase their scale lengths with time as they grow in mass (Barden et al. 2005; Trujillo et al. 2004, 2006), resulting in a constant mass-size relation with cosmic time.

In this context, the study of the interstellar medium (ISM) is crucial to understand the chemical evolution of galaxies, since the enriched material is expelled during the evolution of stars, is mixed with the already existing gas, and condenses to form new stars. The ISM is fundamentally gaseous, and its chemical abundance can be derived by analysing spectroscopic features, that is, nebular emission lines. These emission lines are excited by photoionisation of the interstellar gas by hot and young massive stars (Aller 1984; Osterbrock 1989), which form clouds of ionised hydrogen (H II regions) where star formation (among other processes) takes place. As oxygen is the most abundant heavy element in Universe, this makes it the best proxy for the total gas metallicity.

The study of the Milky Way (MW) is also an exceptional tool for our understanding of galaxy evolution, mainly because we can observe both the stellar and the gaseous components in greater detail than in other galaxies. Among other chemical properties of our Galaxy, the gas abundance gradient has been extensively studied (e.g. Shaver et al. 1983; Deharveng et al. 2000; Pilyugin et al. 2003; Esteban et al. 2005; Quireza et al. 2006; Rudolph et al. 2006; Balser et al. 2011); it is still not properly traced, however, especially in the inner parts. Therefore, complementary information coming from data of external galaxies would help us to overcome this problem.

The study of the gas metallicity in external individual galaxies using spectroscopic data allows us to shed light on fundamental physical properties of galaxies, such as SFR (e.g. Ellison et al. 2008; Lara-López et al. 2010; López-Sánchez 2010), mass and luminosity (e.g. Lequeux et al. 1979; Skillman et al. 1989; Tremonti et al. 2004; Rosales-Ortega et al. 2012), effective yield and rotation velocity (e.g. Vila-Costas \& Edmunds 1992; Garnett 2002; Pilyugin et al. 2004; Dalcanton 2007), or stellar-to-gas fraction (e.g. Zahid et al. 2014; Ascasibar et al. 2015). Moreover, studying these relations at different redshifts can help us to understand the assembly history and evolution of galaxies (e.g. Kobulnicky \& Koo 2000; Maiolino et al. 2008; Mannucci et al. 2009, 2010; Belli et al. 2013). The inside-out scenario is not only supported by studies focused on the stellar content in galaxies. Gas metallicity studies have also been key elements in favour of such disc growth, predicting a relatively quick self-enrichment with oxygen abundances and an almost universal negative metallicity gradient once it is normalised to the galaxy optical size (Boissier \& Prantzos 1999, 2000). Several observational studies have found this radial decrease in the oxygen abundance along the discs of nearby galaxies (e.g. Vila-Costas \& Edmunds 1992; Zaritsky et al. 1994; van Zee et al. 1998; Bresolin et al. 2009; Moustakas et al. 2010; Rich et al. 2012).

However, gas metallicity studies have also presented evidence of the existence of some behaviours in the oxygen abundance profiles that deviate from the pure inside-out scenario: A decrease or a nearly flat distribution of the abundance in the innermost region of discs, first observed by Belley \& Roy (1992); and a flattening in the gradient in the outer regions measured in several works (Martin \& Roy 1995; Vilchez \& Esteban 1996; Roy \& Walsh 1997, among others). These features have been theoretically suggested to be motivated, for instance, by the presence of radial migration (Minchev et al. 2011, 2012). Nevertheless, their origin is still unknown.

All these spectroscopic studies were limited by statistics, either in the number of observed H II regions or in the coverage of these regions across the galaxy surface. The advent of integral field spectroscopy (IFS) techniques offers astronomers the opportunity to overcome these limitations by tracing the distribution of ionised gas and estimating spatially resolved chemical abundances for the gas phase. Its two-dimensional spatial coverage allows us to extract several hundreds or even thousands of spectra across the entire galaxy extent. This enables studying the variation of gas properties throughout the whole disc.

Moreover, IFS surveys offer the opportunity of extending the study to a large number of objects, allowing for meaningful statistical analysis. However, until recently, this technique was rarely used in a survey mode. There were only a few exceptions such as the SAURON survey (Bacon et al. 2001) and the Disk Mass Survey (Bershady et al. 2010).

These pioneering projects were not optimal for a statistical study of the properties of H II regions because they incompletely covered the full extent of the galaxies, among other reasons. Such a statistical study started with the development of the PINGS project (Rosales-Ortega et al. 2010), which acquired IFS mosaic data for a dozen very nearby galaxies. This project was followed by the observation of a larger sample of face-on spiral galaxies (Mármol-Queraltó et al. 2011) as part of the feasibility studies for the CALIFA survey (Sánchez et al. 2012a). The advent of CALIFA allowed extending the study to much more representative samples of nearby galaxies by covering all morphologies.

Based on large-statistics samples of H II regions extracted from galaxies observed by these programs, Sánchez et al. (2012b, 2014) studied the distribution of metals within starforming galaxies and provided the strongest evidence so far for a characteristic gas abundance gradient out to two effective radii $\left(r_{\mathrm{e}}\right)$. These studies also confirmed the behaviours mentioned above that deviate from this gradient, as previously observed by other IFS works on individual galaxies (e.g. Bresolin et al. 2009, 2012; Sánchez et al. 2011; Rosales-Ortega et al. 2011; Marino et al. 2012). However, by selecting H II regions they did not take advantage of the full capability of an IFS study and restricted the study to isolated areas of the galaxies.

Current IFS surveys (e.g. ATLAS ${ }^{3 \mathrm{D}}$, Cappellari et al. 2011; CALIFA, Sánchez et al. 2012a; SAMI, Croom et al. 2012; MaNGA, Bundy et al. 2015) have shown the potential of this 
kind of data to deliver important insights on this and other key questions about the formation and evolution of galaxies at low redshifts. Recent articles have proved the power of this tool to properly map the spatially resolved properties of galaxies by using the full two-dimensional (2D) information spaxel by spaxel that is provided by these surveys (e.g. Papaderos et al. 2013; Singh et al. 2013; Davies et al. 2014; Galbany et al. 2014; Barrera-Ballesteros et al. 2015; Belfiore et al. 2015; Gomes et al. 2016; Ho et al. 2015; Holmes et al. 2015; Li et al. 2015; MartínNavarro et al. 2015; Wilkinson et al. 2015).

In this work, we make use of full 2D information in analysing CALIFA data spaxel by spaxel with the goal of characterising the radial gas abundance profile in a sample of faceon spiral galaxies. We not only focus on the broadly analysed gradient of these profiles, but also study other features that deviate from the simple negative trend, such as inner drops and outer flattenings. We also aim to compare the results with those obtained following the classical procedure of analysing H II regions. A spaxel-by-spaxel study allows us to improve the statistics with respect to previous studies on the topic and also offers the possibility of properly estimating the oxygen distribution across the entire discs over a distance of up to 3-4 disc effective radii. A proper $2 \mathrm{D}$ study of the oxygen abundance distribution that analyses possible azimuthal variations will be presented in a forthcoming work.

The structure of the paper is as follows. Section 2 provides a description of the sample and data we use in this study. Section 3 describes the analysis required to extract the spaxel-wise information. We explain the procedure to detect the H II regions analysed for comparison (Sect. 3.3) and derive the corresponding oxygen abundance values using both methods (Sect. 3.4). Our results are shown in Sect. 4, where we study the oxygen abundance slope distribution (Sect. 4.1), its dependence on different properties of the galaxies (Sect. 4.2), and the existence of a common abundance profile (Sect. 4.3). Finally, the discussion of the results and the main conclusions are given in Sect. 5 .

\section{Data and galaxy sample}

The analysed data were selected from the 939 galaxies that comprise the CALIFA mother sample (Sánchez et al. 2012a). These galaxies were observed using the Potsdam Multi Aperture Spectrograph (PMAS; Roth et al. 2005) at the 3.5m telescope of the Calar Alto observatory with a configuration called PPAK (Kelz et al. 2006). This mode consists of 382 fibres of 2.7 arcsec diameter each, 331 of them (the science fibres) covering an hexagonal field of view (FoV) of $74^{\prime \prime} \times 64^{\prime \prime}$. To achieve a filling factor of $100 \%$ along the full FoV and increase the spatial resolution, a dithering scheme of three pointings was adopted. Two different setups were chosen for the observations: V500, with a nominal resolution of $\lambda / \Delta \lambda \sim 850$ at $5000 \AA$ $(F W H M \sim 6 \AA)$ and a wavelength range from 3745 to $7500 \AA$, and V1200, with a better spectral resolution of $\lambda / \Delta \lambda \sim 1650$ at $4500 \AA(F W H M \sim 2.7 \AA)$ and ranging from 3650 to $4840 \AA$. The data analysed here were calibrated with version 1.5 of the reduction pipeline. More detailed information about the CALIFA sample, observational strategy and data reduction can be found in Sánchez et al. (2012a), Husemann et al. (2013), and GarcíaBenito et al. (2015).

After following the standard steps for fibre-based IFS data reduction, the pipeline provides a regular-grid datacube, with $x$ and $y$ coordinates indicating the right ascension and declination of the target and $z$ being the step in wavelength for all galaxies in the sample. An inverse-distance weighted image reconstruction scheme was performed as interpolation method to reconstruct the datacube. As a result, we have individual spectra for each sampled spaxel of $1^{\prime \prime} \times 1^{\prime \prime}$ and a final spatial resolution for the datacubes of $F W H M \sim 2.5$ arcsec.

The subset of galaxies used in this work was selected by adopting the following criteria:

(a) Spiral galaxies with morphological types between $\mathrm{Sa}$ and $\mathrm{Sm}$, including barred galaxies.

(b) Face-on galaxies, with $i<60^{\circ}$, to avoid uncertainties induced by inclination effects.

(c) Galaxies with no evident signatures of interaction or merging (i.e. tails, bridges, rings, etc.).

(d) Galaxies with $\mathrm{H} \alpha$ detected along different galactocentric distances with a signal-to-noise ratio $(\mathrm{S} / \mathrm{N})$ for the spaxels above 4 on average.

The classification according to morphological type and into interacting or non-interacting galaxy was based on the visual inspection carried out by Walcher et al. (2014, see details in the article). After imposing these restrictions, the galaxy sample was reduced to 204 galaxies. From these, we only analysed the 129 galaxies that have finally been observed by the CALIFA collaboration with the V500 setup.

Figure 1 shows the comparison of the distribution of morphological types, absolute $B$-band magnitudes, and disc effective radii between the spiral galaxies in the CALIFA mother sample and the sample used in this study. There is a clear deficiency of earlier (Sa-Sab) and later (Sc-Sdm) spirals; the sample is dominated by intermediate galaxies. This may be due to the imposition of ionised gas throughout the discs, which we have prioritised to perform a detailed 2D study of the gas metallicity. The distribution of galaxies according to their absolute magnitude clearly shows an absence of faint galaxies, with values above -18 mag. This fact is a consequence of a selection effect in the definition of the CALIFA mother sample (Walcher et al. 2014). The CALIFA mother sample was created by applying a size selection criterion defined by a minimum apparent isophotal size. A size-limited sample like this favours inclined over faceon sytems because the inclination increases the apparent isophotal size (because the surface brightness increases). This effect causes these inclined galaxies to dominate the low-luminosity population of galaxies. Because we selected only face-on galaxies with $i<60^{\circ}$, we automatically discarded all these faint galaxies. On the other hand, a correlation between the morphological type of the galaxies and the mass (thus, with the luminosity, see e.g. Roberts \& Haynes 1994; González Delgado et al. 2015) has been found, where later types present lower masses (and luminosities). This contributes to the deficiency of later spirals that is found in the sample because of the lack of low-luminosity galaxies and the correlation between these two parameters. For the distribution of galaxies according to their disc effective radius, we finally obtained similar distributions for the CALIFA spiral galaxies and our sample: the sample is dominated by galaxies with $r_{\mathrm{e}}$ between 4 and $10 \mathrm{kpc}$. Furthermore, there are no significant differences in the distribution when the galaxies are separated into barred and non-barred galaxies, galaxies of all sizes are present in both cases.

Figure 2 shows the distribution of our sample (filled dots) and the total sample of CALIFA spiral galaxies (empty dots) along the $(B-V)$ vs. $M_{V}$ colour-magnitude diagram. Our sample covers the same range as the CALIFA spirals above 

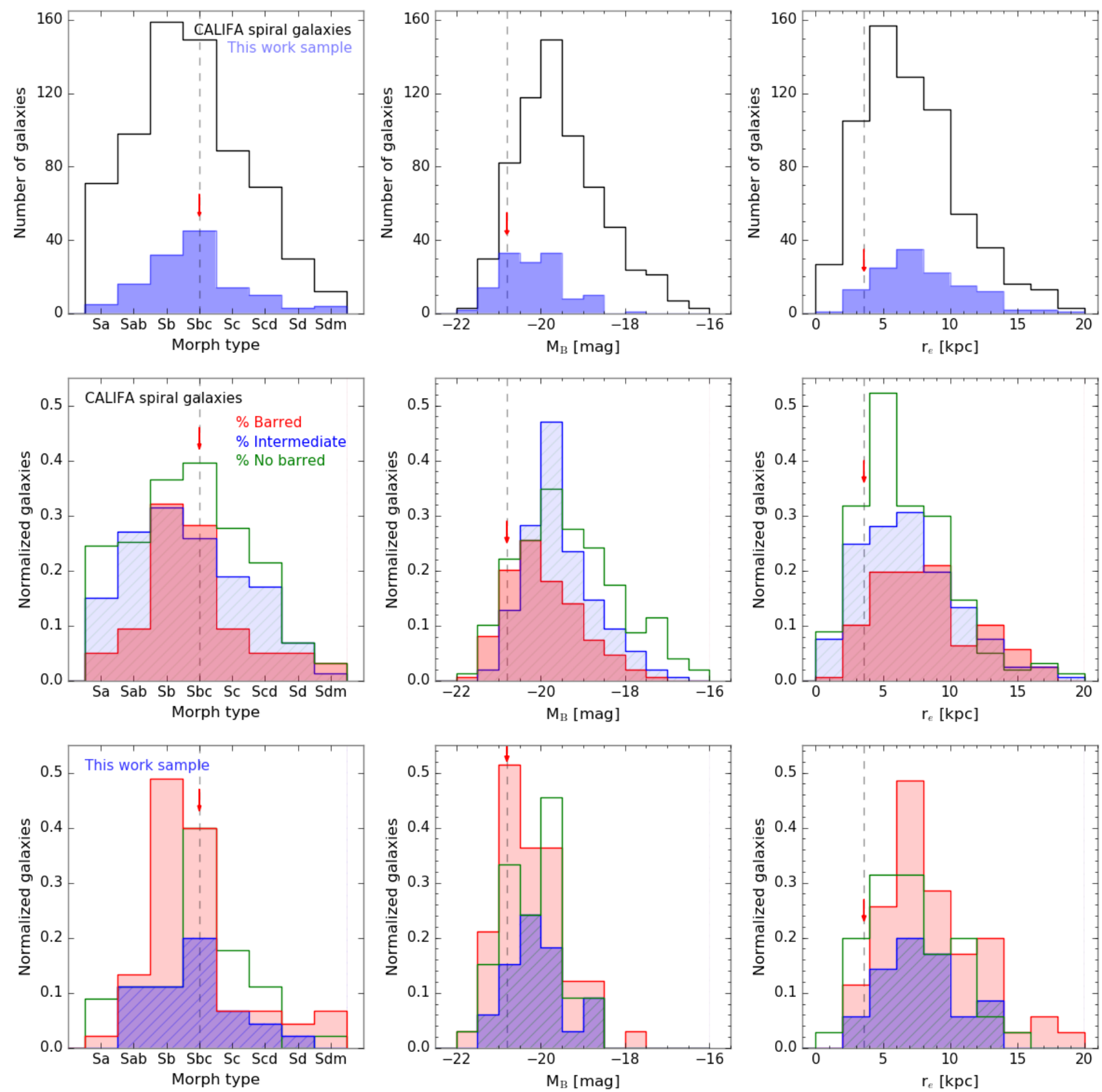

Fig. 1. Distribution of morphological types (top left), absolute $B$-band magnitudes (top middle), and disc effective radii (top right) of the spiral galaxies in the CALIFA mother sample (unfilled black histograms) and the galaxies selected in this work (filled blue histograms). The middle and bottom panels show the normalised distributions separated according to the presence or absence of bars: barred galaxies (red histograms), unbarred galaxies (green histograms) and not clearly identified galaxies (blue histograms). The dashed grey line and the red arrow mark the location of the MW in each panel. The values for the absolute $B$-band magnitude $\left(M_{B}=-20.8 \mathrm{mag}\right)$ and the disc effective radius $\left(r_{\mathrm{e}}=3.6 \mathrm{kpc}\right)$ are taken from Karachentsev et al. (2004) and Bovy \& Rix (2013, considering $\left.r_{\mathrm{e}} \sim 1.67 r_{\mathrm{d}}\right)$ respectively.

$M_{V} \sim-17$ mag, excluding the faint galaxies below this limit for the reasons explained above.

We note that with the limitations we mentioned, which are linked to the criteria we adopted to define the sample, the selected set of galaxies is well suitable to carry out the study presented here, that is, a detailed 2D study of the radial gas metallicity distribution in spiral galaxies.

\section{Analysis}

The main goal of this study is to characterise the radial abundance profiles in the galaxy sample using the full 2D information and compare it with the results obtained using only the H II regions. In this section we describe the procedure followed to select the spaxels, analyse their individual spectra, and to derive the corresponding oxygen abundance. We also explain how we 


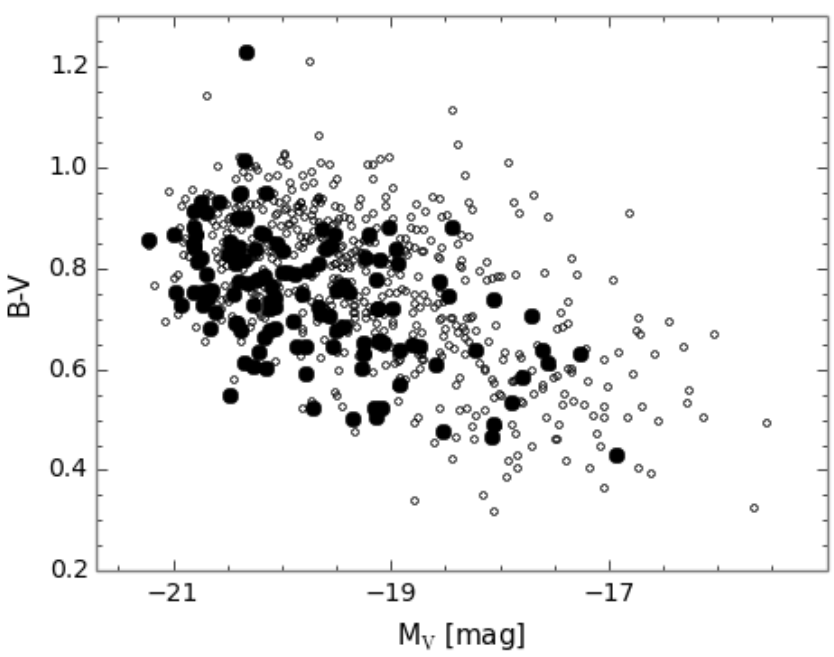

Fig. 2. Distribution of the spiral galaxies in the CALIFA mother sample (empty small dots) and the galaxies selected in this work (filled large dots) in the $(B-V)$ vs. $M_{V}$ colour-magnitude diagram.

have detected the H II regions used for comparison and the subsequent analysis.

\subsection{Measurement of the emission lines}

In the spectrum of a galaxy (or a portion of it), the emission lines are superimposed on the underlying stellar spectrum. To accurately measure the emission line fluxes, the stellar contribution must be estimated and subtracted from the galaxy spectrum to derive a pure gas spectrum (allowing for the contribution of noise from the stellar populations) for each individual spaxel (or H II region).

Several tools have been developed to model the underlying stellar population and decouple it from the emission lines (e.g. Cappellari \& Emsellem 2004; Cid Fernandes et al. 2005; Ocvirk et al. 2006; Sarzi et al. 2006; Koleva et al. 2009; Sánchez et al. 2011). Most of them are based on the assumption that the SFH of a galaxy can be approximated as a sum of discrete star formation bursts and, therefore, that the stellar spectrum can be considered as the result of the combination of spectra of different simple stellar populations (SSP) with different ages and metallicities.

In this work, we made use of a fitting package named FIT3D ${ }^{1}$ to model both the continuum emission and the emission lines. This tool uses an SSP template grid that comprises 156 individual populations covering 39 stellar ages between 0.001 and 14.1 Gyr and four metallicities between 0.004 and 0.03 . This grid combines the Granada models from González Delgado et al. (2005) for $t<63$ Myr with those provided by the MILES project (Vazdekis et al. 2010; Falcón-Barroso et al. 2011) for older ages (following Cid Fernandes et al. 2013). This way, FIT3D fits each spectrum by a linear combination of the SSP templates that are collected in the library after correcting for the appropriate systemic velocity and velocity dispersion (including the instrumental dispersion, which dominates the total observed dispersion) and taking into account the effects of dust attenuation. We adopted the Cardelli et al. (1989) law for the stellar dust extinction with $R_{V}=3.1$.

To measure the emission line fluxes and after the stellar component is subtracted, FIT3D performs a multi-component fitting using a single Gaussian function per emission line plus

http://www . astroscu. unam.mx/ sfsanchez/FIT3D a low-order polynomial function. When more than one emission line was fitted simultaneously (e.g. for doublets and triplets like the [N II] lines), the systemic velocity and velocity dispersion were forced to be equal to decrease the number of free parameters and increase the accuracy of the deblending process. The measured line fluxes include all lines required in determining the gas metallicity using strong-line methods, that is, $\mathrm{H} \alpha, \mathrm{H} \beta,[\mathrm{O}$ II] $\lambda 3727$, [O III] $\lambda 4959$, [O III] $\lambda 5007$, [N II] $\lambda 6548$, [N II] $\lambda 6584$, [S II] $\lambda 6717$, and [S II] $\lambda 6731$. FIT3D provides the intensity, equivalent width (EW), systemic velocity, and velocity dispersion for each emission line. The statistical uncertainties in the measurements were calculated by propagating the error associated with the multi-component fitting and taking into account the $\mathrm{S} / \mathrm{N}$ at the spectral region.

As indicated above, FIT3D fits both the underlying stellar population and the emission lines. In addition to the parameters derived for the emission lines, the fitting algorithm therefore provides information related to the stellar population: the luminosity-weighted ages and metallicities, the average dust attenuation, the mass-weighted ages and metallicities, the average mass-to-light ratio, and the individual weights of the multi-SSP decomposition that in essence trace the SFH.

The entire procedure of fitting and subtracting the underlying stellar population and measuring the emission lines using FIT3D is described in more detail in Sánchez et al. (2011) and Sánchez et al. (2015b).

We note that all these parameters (both stellar and gas) were derived spaxel by spaxel for each individual spectrum of the datacubes, providing the sets of 2D maps that are the base of our analysis.

\subsection{Extracting information spaxel by spaxel}

As a result of the FIT3D fitting process, we obtained the set of 2D intensity maps for the emission lines that are required to determine the gas metallicity. To guarantee realistic measurements of the emission line fluxes for each spaxel, we adopted a lower limit below which we considered that the fluxes are of the same order as the continuum error. In this way, we discarded the spaxels whose emission line fluxes employed in the determination of the oxygen abundance are lower than $1 \sigma$ over the continuum level. From all the spaxels with flux values above this limit, we now selected those that are associated with star formation (SF).

The intensities of strong lines were broadly used to discern between different types of emission according to their main excitation source (i.e. starburst or AGN) throughout the socalled diagnostic diagrams (e.g. Baldwin et al. 1981; Veilleux \& Osterbrock 1987). In most cases these diagrams are very useful in distinguishing between strong ionisation sources, such as classical H II/SF regions and powerful AGNs. However, they are less accurate in distinguishing between low-ionisation sources, such as weak AGNs, shocks, and/or post-AGBs stars (Stasińska et al. 2008; Cid Fernandes et al. 2011). Alternative methods based on a combination of the classical line ratios and additional information regarding the underlying stellar population have been proposed, for instance, the so-called WHAN diagram (Cid Fernandes et al. 2011). This diagram uses the $E W(\mathrm{H} \alpha)$ to take into account weak AGNs and 'retired' galaxies, that is, galaxies that have stopped forming stars and are ionised by hot low-mass evolved stars.

The most commonly used diagnostic diagram was proposed by Baldwin et al. (1981, hereafter BPT diagram). This diagram makes use of the $[\mathrm{N} \mathrm{II}] \lambda 6584 / \mathrm{H} \alpha$ and $[\mathrm{O}$ III] $\lambda 5007 / \mathrm{H} \beta$ line ratios, which are less affected by dust attenuation because of their 
proximity in wavelength space. Different demarcation lines for BPT diagram have been proposed to distinguish between SF regions and AGNs. The most popular are the Kauffmann et al. (2003) and Kewley et al. (2001) curves. Pure H II /SF regions are considered to be below the Kauffmann et al. (2003) curve and AGNs above the Kewley et al. (2001) curve. The area between the two curves is broadly and erroneously assigned to a mixture of different ionisation sources, since pure SF regions can also be found here.

These two demarcation lines have a different origin. The Kewley et al. (2001) curve was derived theoretically using photoionisation models and corresponds to the maximum envelope for ionisation produced by OB stars. The Kauffmann et al. (2003) curve has an empirical origin, based on the analysis of the emission lines for the integrated spectra of SDSS galaxies. It describes the envelope for classical H II /SF regions well that are found in the discs of late-type spiral galaxies. However, it excludes certain kinds of SF regions that have already been found above this demarcation line (Kennicutt et al. 1989; Ho et al. 1997 and; more recently, Sánchez et al. 2014). Selecting H II/SF regions based on the Kauffmann et al. (2003) curve may therefore bias our sample towards classical disc regions. Moreover, it does not guarantee that other sources of non-stellar ionisation are excluded that might populate this area, such as weak AGNs, shocks, and/or post-AGBs stars. We adopted the Kewley et al. (2001) curve to exclude strong AGNs and an EW criterion to exclude weak AGNs and "retired" emission (Cid Fernandes et al. 2011). However, we were more restrictive in the EW range than Cid Fernandes et al. (2011) and established the limit in $6 \AA$ to also guarantee a better $\mathrm{S} / \mathrm{N}$ of the emission lines for all spaxels. For a detailed analysis of the weak emission procedure spaxel by spaxel using CALIFA data, see Gomes et al. (2016).

In addition to the light from the ionised SF regions, there is a background of diffuse nebular emission that extends over the whole disc of the galaxies and can blur contribution of the SF regions, which is the subject of our study. However, most of the diffuse ionised emission has been excluded by the $1 \sigma$ limit imposed to the flux of the selected spaxels and the EW criterion, since this emission is dominated by the stellar continuum. For a comparison of the location in the BPT diagram for low-ionisation emission sources see, for instance, Kehrig et al. (2012), Papaderos et al. (2013), and Gomes et al. (2016).

The top left panel of Fig. 3 shows the [O III] $\lambda 5007 / \mathrm{H} \beta$ vs. [N II] $\lambda 6584 / \mathrm{H} \alpha$ diagnostic diagram for the spaxels in all 129 galaxies of our sample above the considered flux limit and with $E W(\mathrm{H} \alpha)>6 \AA$. The solid and dashed lines represent the Kewley et al. (2001) and Kauffmann et al. (2003) demarcation curves, respectively. Some points dominated by SF ionisation might be present above the Kewley et al. (2001) curve as a result of the errors of the considered emission lines. They are, therefore, excluded from further analysis by our criteria for selecting spaxels associated with SF activity. However, the spaxels that present larger errors are those with a low $\mathrm{S} / \mathrm{N}$, and they do not affect our conclusions significantly.

The top right panel of Fig. 3 shows the location of the selected spaxels in a particular spiral galaxy of the sample, NGC 0165 , over-plotted to the $\mathrm{H}_{\alpha}$ map. Blue dots correspond to the spaxels classified as SF regions and red dots are those that lie higher than the Kewley et al. (2001) curve and can therefore be associated with AGNs. The figure shows that the selected spaxels follow the $\mathrm{H}_{\alpha}$ emission. The classification of red points as ionised by AGNs in this galaxy as well as in other cases is clearly false because of their distance to the galactic centres.
This misclassification is most probably due to the errors in the considered emission lines. As in the previous case, they could cause some spaxels that are dominated by SF ionisation to lie higher than the Kewley et al. (2001) curve in a similar way that errors could produce the opposite effect with spaxels associated to AGNs. As these spaxels represent only the $2 \%$ for the whole sample, including them would not alter our results significantly, and thus they were discarded from the further analysis. For this galaxy, 1201 spaxels were associated with SF regions.

\subsection{Detection and selection of H II regions}

We detected the $\mathrm{HII}$ regions and extracted the corresponding spectra using a semi-automatic procedure named HIIEXPLORER ${ }^{2}$. The procedure is based on two assumptions: (a) $\mathrm{H}$ II regions are peaky and isolated structures with a strongly ionised gas emission, particularly $\mathrm{H} \alpha$, that is significantly higher than the stellar continuum emission and the average ionised gas emission across the galaxy; (b) H II regions have a typical physical size of about one hundred or a few hundred parsecs (González Delgado \& Pérez 1997; Oey et al. 2003; Lopez et al. 2011), which corresponds to a typical projected size of a few arcsec at the standard distance of the galaxies in the sample.

A more detailed description of this algorithm can be found in Sánchez et al. (2012b), with a few modifications presented in Sánchez et al. (2015a). Basically, the main steps of the process are as follows: (i) first we create a narrow-band image of $120 \AA$ width centred on $\mathrm{H} \alpha$ shifted at the redshift of each galaxy; (ii) this image is used as an input for HIIEXPLORER. The algorithm detects the brightest pixel in the map and then adds all the adjacent pixels up to a distance of $3.5^{\prime \prime}$ if their fluxes exceed $10 \%$ of the peak intensity. After the first region is detected and separated, the corresponding area is masked from the input image and the procedure is repeated until no peak with a flux exceeding the median $\mathrm{H} \alpha$ emission flux of the galaxy is found. The result is a segmentation FITS file describing the pixels associated with each detected H II region. Finally; (iii) the integrated spectrum corresponding to each segmented region is extracted from the original datacube, and the corresponding position table of the detected area is provided.

After we extracted the spectra for the detected clumpy ionised regions, we applied the same analysis described in Sects. 3.1 and 3.2: each extracted spectrum was decontaminated by the underlying stellar population using FIT3D, and the emission line fluxes were measured by fitting each line with a Gaussian function. These line ratios were used to distinguish between the detected ionised regions, the ones associated with star formation. In a similar way as for individual spaxel spectra, using the BPT diagram H II/SF regions were considered to be under the Kewley et al. (2001) curve and present an $E W(\mathrm{H} \alpha)>6 \AA$.

Figure 3 (bottom left) shows the $[\mathrm{O}$ III $] \lambda 5007 / \mathrm{H} \beta$ vs. [N II] $\lambda 6584 / \mathrm{H} \alpha$ diagnostic diagram for the $\mathrm{H}$ II $/ \mathrm{SF}$ regions. The solid and dashed lines represent the Kewley et al. (2001) and Kauffmann et al. (2003) demarcation curves, respectively.

Figure 3 (bottom right) shows an example of an $\mathrm{H} \alpha$ map for one spiral galaxy of the sample, NGC 0165, where the location of the H II regions is superimposed as black segmented contours. For this galaxy we detected $72 \mathrm{H}$ II regions.

http://www. astroscu. unam.mx/ sfsanchez/HII_explorer 

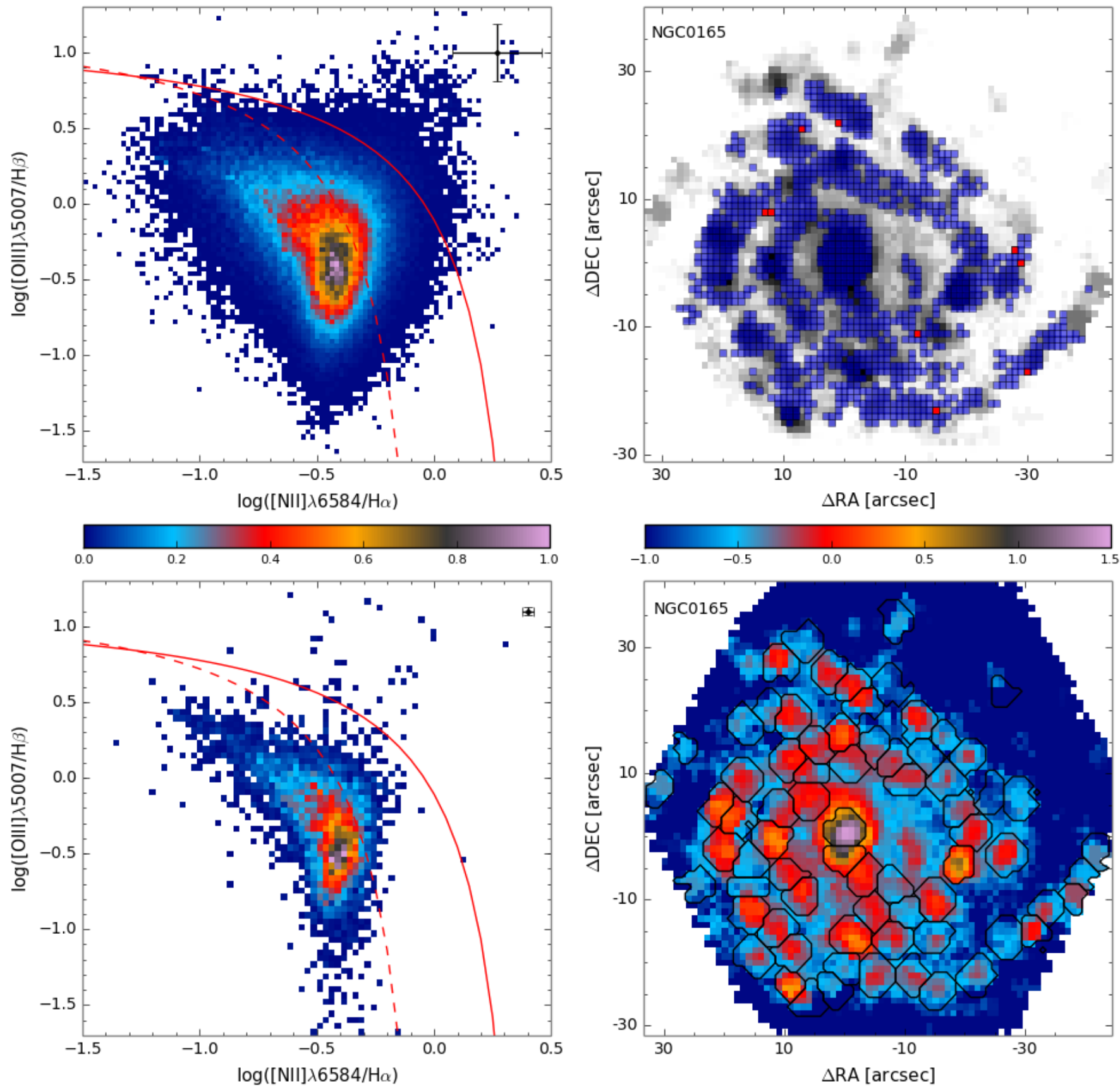

Fig. 3. Left panels: normalised density distribution of the spaxels with $\mathrm{EW}(\mathrm{H} \alpha)$ above $6 \AA$ (top) and of the detected $\mathrm{H}$ II/SF regions (bottom) along the BPT diagram. The solid and dashed lines in both panels represent the Kewley et al. (2001) and Kauffmann et al. (2003) demarcation curves. SF regions are considered to be below the Kewley et al. (2001) curve. Right panels: location of the spaxels classified as SF regions (blue dots) and AGNs (red dots) according to the BPT diagram superimposed on the IFS-based H $\alpha$ map derived for one galaxy of the sample, NGC 0165 (top) and a $\mathrm{H} \alpha$ map in units of $(\log 10) 10^{-16} \mathrm{erg} \mathrm{s}^{-1} \mathrm{~cm}^{-2} \operatorname{arcsec}^{-1}$ for NGC 0165 , together with the detected H II regions shown as black segmented contours (bottom).

\subsection{Measurement of the oxygen abundances}

A direct procedure to measure abundances from observed spectra requires using temperature-sensitive line ratios such as [O III] $\lambda \lambda 4959,5007 /[\mathrm{O}$ III] $\lambda 4363$. This is known as the $T_{\mathrm{e}}$-method (Peimbert \& Costero 1969; Stasińska 1978; Pagel et al. 1992; Vilchez \& Esteban 1996; Izotov et al. 2006). However, some of these auroral or nebular lines are very faint, and they become even fainter as the metallicity increases (when a more efficient cooling mechanism begins to act through the metal lines, which produces a decrease in the temperature), and eventually, they are too weak to be detected. Furthermore, as a result of the weakness of the involved lines, the $T_{e}$-method can only be applied to nearby and low-metal objects for which very high $\mathrm{S} / \mathrm{N}$ spectra are observable.

It is therefore necessary to look for indirect methods that allow us to estimate the chemical abundances. To do this, abundance indicators based on the relations between metallicity and the intensity of strong and more readily observable lines have been developed. These methods have first been proposed by 
Alloin et al. (1979) and Pagel et al. (1979). Since then, several calibrators based on direct estimations of oxygen abundances (Zaritsky et al. 1994; Pilyugin 2000; Denicoló et al. 2002; Pettini \& Pagel 2004; Pérez-Montero \& Díaz 2005; Pilyugin $\&$ Thuan 2005; Pilyugin et al. 2010; Marino et al. 2013) and photoionisation models (Dopita \& Evans 1986; McGaugh 1991; Kewley \& Dopita 2002; Kobulnicky \& Kewley 2004; Dopita et al. 2006, 2013; Pérez-Montero 2014) have been proposed and are widely used todays. For a revision of the different methods, their strengths and their caveats, see López-Sánchez et al. (2012).

In this work we aim to derive the spatial distribution of the oxygen abundance across the considered galaxies. For this purpose, we used the emission line intensities derived spaxel by spaxel for the sample of $\mathrm{H}$ II regions described before. We adopted the empirical calibrator based on the O3N2 index that was first introduced by Alloin et al. (1979):

$\mathrm{O} 3 \mathrm{~N} 2=\log \left(\frac{[\mathrm{O} \mathrm{III}] \lambda 5007}{\mathrm{H} \beta} \times \frac{\mathrm{H} \alpha}{[\mathrm{N} \mathrm{II}] \lambda 6584}\right)$.

This index: (i) is only weakly affected by dust attenuation because of the close distance in wavelength between the lines in both ratios; (ii) presents a monotonic dependence on the abundance and (iii) uses emission lines covered by CALIFA wavelength range. One of the most popular calibrations for this index has been proposed by Pettini \& Pagel (2004, hereafter PP04). However, this indicator lacks observational points at the high-metallicity regime $(12+\log (\mathrm{O} / \mathrm{H})>8.2)$ and instead uses predictions from photoionisation models. Therefore, we here adopted the improved calibration proposed by Marino et al. (2013, hereafter M13), where $12+\log (\mathrm{O} / \mathrm{H})=8.533-0.214 \times \mathrm{O} 3 \mathrm{~N} 2$. This calibration uses $T_{\mathrm{e}}$-based abundances of $\sim 600 \mathrm{H}$ II regions from the literature together with new measurements from the CALIFA survey, providing the most accurate calibration to date for this index. The derived abundances have a calibration error of $\pm 0.08 \mathrm{dex}$, and the typical errors associated with the pure propagation of the errors in the measured emission lines are about 0.05 dex.

\subsection{Oxygen abundance gradients}

To derive the radial distribution of the oxygen abundance for each galaxy, we determined the position angle and ellipticity of the disc to obtain the deprojected galactocentric distances of the selected spaxels. These morphological parameters were derived by performing a growth curve analysis (Sánchez et al. 2014, hereafter S14). The inclination was deduced by also assuming an intrinsic ellipticity for galaxies of $q=0.13$ (Giovanelli et al. 1994):

$\cos ^{2} i=\frac{(1-\epsilon)^{2}-q^{2}}{1-q^{2}}$,

where $\epsilon$ is the ellipticity provided by the analysis and given by $\epsilon=1-b / a$, with $a$ and $b$ being the semi-major and semi-minor axes. We preferred not to correct for the inclination effects in galaxies with an inclination below $35^{\circ}$ because the uncertainties in the derived correction exceed the very small effect on the spatial distribution of the spaxels, even more when an intrinsic ellipticity is also considered.

We then derived the galactocentric distance for each spaxel, which was later normalised to the disc effective radius, as suggested in Sánchez et al. (2012b, 2013). This parameter was derived from the disc scale-length based on an analysis of the azimuthal surface brightness profile (SBP), explained in Appendix A of S14. Other normalisation length-scales were used for better comparison with other studies, such as the $r_{25}$ radius, which is defined as the radius corresponding to a surface brightness level of $25 \mathrm{mag} / \mathrm{arcsec}^{2}$ in the SDSS $r$-band ${ }^{3}$, and the physical scale of the galaxy, that is, the distances in kpc.

Finally, we obtained the radial distribution of the oxygen abundance for each galaxy. To characterise this profile, we performed an error-weighted linear fit to the derived oxygen abundance mean values for radial bins of $0.25 r_{\mathrm{e}}$ within the range between 0.5 and $2.0 r_{\mathrm{e}}$. The radial binning was made to minimise possible azimuthal differences in the oxygen abundance distribution, and the size of the bins was chosen to match the seeing value. We excluded the innermost region $\left(r / r_{\mathrm{e}}<0.5\right)$, which sometimes presents a nearly flat distribution or even a drop towards the centre (e.g. Belley \& Roy 1992; Rosales-Ortega et al. 2011; Sánchez et al. 2012b, 2014). We also omitted the outer region $\left(r / r_{\mathrm{e}}>2.0\right)$, which it is found to have a flattening in the abundance gradient for galaxies covering regions beyond $r_{25}$ (e.g. Martin \& Roy 1995; Vilchez \& Esteban 1996; Roy \& Walsh 1997; van Zee et al. 1998; Bresolin et al. 2009, 2012; Rosales-Ortega et al. 2011; Marino et al. 2012; López-Sánchez et al. 2015). The edges of the range were obtained based on a visual inspection of each individual galaxy, discarding the regions where we observed the mentioned features. The fitted interval has changed with respect to S14, simply because of a better space coverage that allowed us to refine the radial limits. The linear fit was weighted using the standard deviations of (mean) values within each bin and considered only the bins that contained at least nine values of the oxygen abundance. This minimum number of values required for each bin was determined to ensure a precision in the derived mean that is ten times better than the dynamic range of abundance values covered in the fit, taking into account the dispersion in the measurements.

It is important to note that uncertainties in the determination of the deprojected galactocentric distances can significantly affect the derivation of the abundance gradients. Moreover, as the radial galactocentric distances are normalised to the disc effective radius, the uncertainties in the determination of the $r_{\mathrm{e}}$ can also contribute to the scatter obtained on the final oxygen gradients. On one hand, performing Monte Carlo simulations, we obtained that an error of $5^{\circ}$ in the inclination and PA of the galaxies can produce a dispersion in the gradient distribution of at most $0.05 \mathrm{dex} / r_{\mathrm{e}}\left(0.02 \mathrm{dex} / r_{\mathrm{e}}\right.$ on average $)$. On the other hand, comparing different methods to derive the $r_{\mathrm{e}}$ (as described in S14) and taking into account both the nominal errors and the differences among them, the overall contribution to the dispersion in the gradient distribution coming from the derivation of the $r_{\mathrm{e}}$ is at most $0.04 \mathrm{dex} / r_{\mathrm{e}}\left(0.01 \mathrm{dex} / r_{\mathrm{e}}\right.$ on average $)$. All these uncertainties are well accounted for by our error estimation of the gradient (0.05 dex $/ r_{\mathrm{e}}$, see Sect. 4.1).

In Fig. 4 we present an example of the abundance gradient for the same galaxy shown in the right panels of Fig. 3, NGC 0165, using both the spaxel-wise information (colour map) and the individual H II regions (violet dots). The diamonds represent the mean oxygen abundance values of the radial bins, with the error bars indicating the corresponding standard deviations. The red solid line is the error-weighted linear fit derived for values within the range between 0.5 and $2.0 r_{\mathrm{e}}$ (yellow diamonds), and the dashed black line is the linear regression for the individual $\mathrm{H}$ II regions. This figure illustrates the procedure

Using the seventh data release (DR7, Abazajian et al. 2009). 


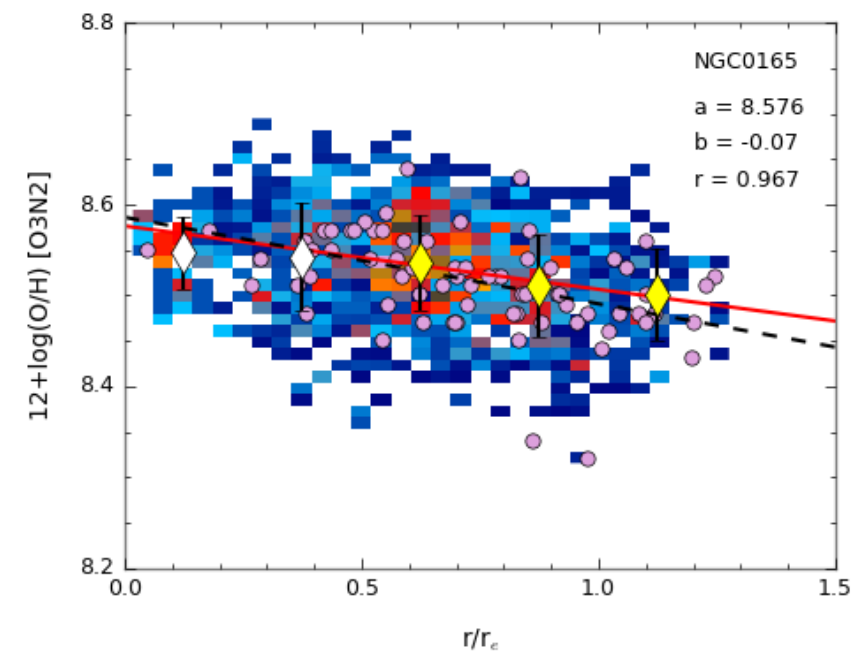

Fig. 4. Radial density distribution of the spaxels in the oxygen abundance-galactocentric distance space for the same galaxy as in Fig. 3 (right panels). The radial distances are deprojected and normalised to the disc effective radius. The diamonds represent the mean oxygen abundance values, with the error bars representing the corresponding standard deviations, for bins of $0.25 r_{\mathrm{e}}$ and the red solid line the errorweighted linear fit derived for values within the range between 0.5 and $2.0 r_{\mathrm{e}}$ (yellow diamonds). The parameters of the fit are shown in the upper right corner of the panels, including the zero point (a), the slope (b) and the correlation coefficient (r). The violet dots correspond to the oxygen abundances derived for the individual H II regions, and the dashed black line is the linear regression for these points.

explained before to derive the oxygen abundance gradient. From the original 129 galaxies, we were able to fit 122 lines, and the remaining galaxies were discarded from further analysis because of the low number of spaxels associated with SF regions that are needed to carry out the linear fit. This final sample provides more than 185000 oxygen abundance values, $\sim 8230$ of them beyond two disc effective radii, and with more than $7100 \mathrm{H}$ II regions to compare with, $\sim 605$ beyond $2 r_{\mathrm{e}}$.

\section{Results}

With the procedure explained in the previous section we obtained the oxygen abundance gradient for the 122 galaxies in our sample. We describe the main properties of these abundance profiles below.

\subsection{Abundance gradient distribution}

Figure 5 shows the distribution of the correlation coefficient, zero-point, and slope of the abundance gradients for the final sample using both the spaxel-wise information (red dashed histogram) and the individual H II regions (blue filled histogram).

We focus first on the spaxel-wise analysis. For almost all galaxies the correlation coefficient is larger than 0.75 . If we perform a Student's t-test to check the significance of the correlation, we obtain that for $\sim 80 \%$ of the galaxies the oxygen abundance and the radial distance (normalised to the disc effective radius) are significantly correlated to the $95 \%$ level $(0.05)$.

The distribution of zero points ranges between 8.4 and 8.7 , reflecting the mass-range covered by the sample as a consequence of the well-known $\mathcal{M}-\mathcal{Z}$ relation (e.g. Tremonti et al. 2004; Sánchez et al. 2013). The presence of a peak in the distribution and a small standard deviation is the result of a bias in the selection of the sample, explained in Sect. 2, which is due to a lack of low-luminosity galaxies.

Finally, the distribution of slopes presents a characteristic value of $\alpha_{\mathrm{O} / \mathrm{H}}=-0.07 \mathrm{dex} / r_{\mathrm{e}}$ with a standard deviation of $\sigma \sim 0.05 \mathrm{dex} / r_{\mathrm{e}}$. We performed a Lilliefors test (Lilliefors 1967) to assess the compatibility of the distribution with a Gaussian, obtaining a test statistics of 0.07 and a $P$-value of 0.62 , showing that the distribution of slopes has a clear peak and is remarkably symmetric. We ran a Monte Carlo simulation to estimate the contribution of the errors in the derived slopes to the $\sigma$ of the distribution, obtaining that these errors can only explain $49 \%$ of the width distribution. We may have underestimated the errors involved in the determination of the slopes, particularly the effect of the inclination. Otherwise, the remaining $\sigma$ must have another origin that we investigate in more detail in Sect. 4.2.

The analysis for the individual H II regions leads us to very similar results. In this case, we have a wider distribution for the correlation coefficients, but we have to note that the number of points involved in the linear fit is larger using all the individual $\mathrm{H}$ II regions, since we did not apply any kind of radial binning to the data. The correlation coefficient is larger than 0.32 for $\sim 60 \%$ of the galaxies, which corresponds to a significance level of $98 \%(0.02)$. The distribution of zero points covers almost the same range as for the spaxels (between 8.3 and 8.8), allowing us to draw the same conclusions. Finally, the distribution of slopes presents a characteristic value of $\alpha_{\mathrm{O} / \mathrm{H}}=-0.05 \mathrm{dex} / r_{\mathrm{e}}$ with a standard deviation of $\sigma \sim 0.06 \mathrm{dex} / r_{\mathrm{e}}$. The Lilliefors test gives a test statistic of 0.08 and a $P$-value of 0.28 , very similar to the one described for the spaxel-wise analysis. The Monte Carlo simulation yields a contribution of $44 \%$ of the errors in the derived slopes to the distribution width, again insufficient to explain the $\sigma$ of the distribution.

If we use different scales to normalise the radial distances like $r_{25}$ and the physical scale of the galaxy (radius in kpc) for both the spaxel-wise and the individual H II region analysis, we obtain in all cases a similar and narrow distribution, although for the physical scale the distribution is clearly asymmetric, with a tail towards large slopes. We note that our range of masses is narrow, and consequently, so is the range of $r_{\mathrm{e}}$ and $r_{25}$, which in turn causes the distribution when normalising to the physical scale narrow as well, in contrast to what we should obtain for a wider range of masses. The different slope values are given in Table 1.

\subsubsection{Comparison with other calibrators}

It is beyond the purpose of this work to make a detailed comparison of the oxygen abundance gradients derived using different empirical calibrators. However, we compare our results with those provided with other methods by deriving the oxygen radial distributions using some of the most commonly used empirical calibrators: (i) the technique proposed by PP04 for the O3N2 index; (ii) the Pilyugin et al. (2010, hereafter P10) calibration for the ONS index; (iii) and the Dopita et al. (2013, hereafter D13) calibration based on the MAPPINGS IV code developed by the authors.

The PP04 calibration for the O3N2 index, as already mentioned in Sect. 3.4, is one of the most popular calibrations used for this index and is defined as $12+\log (\mathrm{O} / \mathrm{H})=8.73-0.32 \times$ $\mathrm{O} 3 \mathrm{~N} 2$. This calibration is not valid in the low-metallicity regime $(12+\log (\mathrm{O} / \mathrm{H})<8)$, but as we do not reach this limit, this effect will not affect our results. The P10 ONS calibration makes uses of the $N_{2} / R_{2}$ and $S_{2} / R_{2}$ ratios (defined as $R_{2}=[\mathrm{O}$ II $](\lambda 3727+\lambda 3729), N_{2}=[\mathrm{N} \mathrm{II}](\lambda 6548+\lambda 6584)$, 


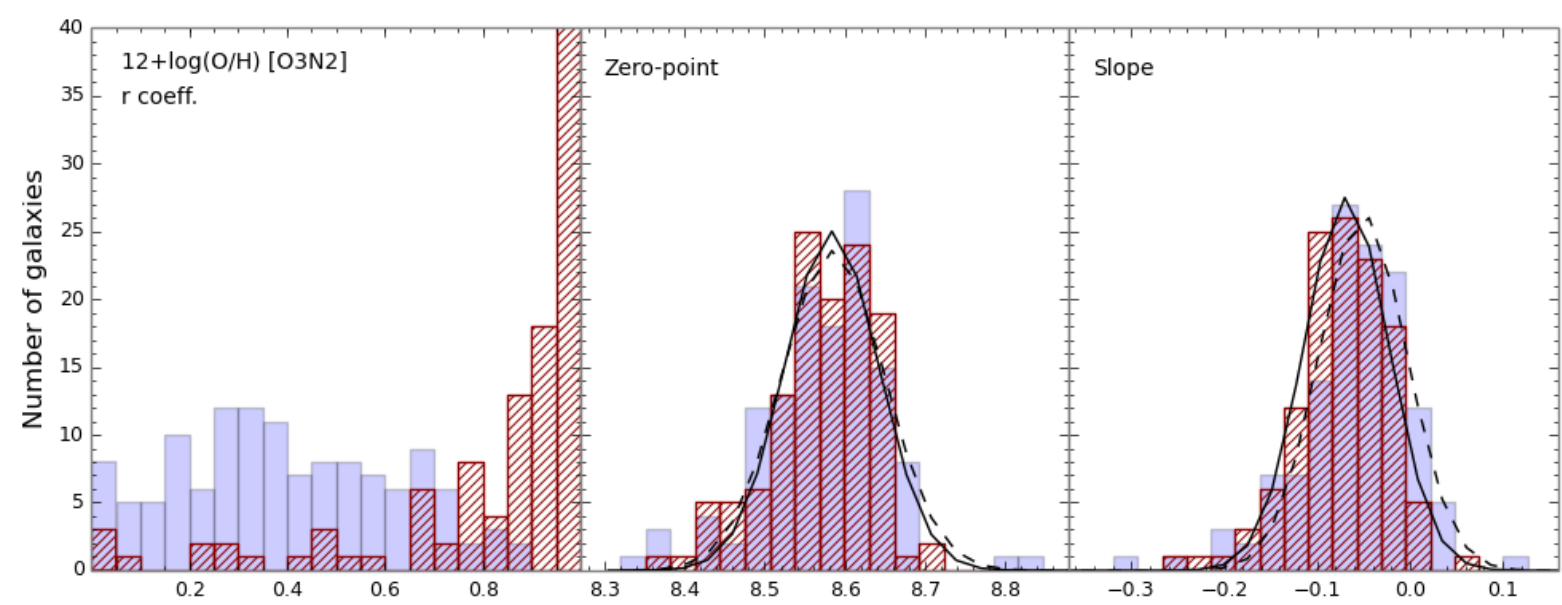

Fig. 5. Distribution of correlation coefficients (left panel), zero points (middle panel), and slopes (right panel) of the linear fits derived for the oxygen abundance gradients of the final sample using spaxel-wise information (dashed red bars) and the individual $\mathrm{H}$ II regions (filled blue bars). For both the zero point and slope distributions the lines represent the Gaussian distribution of the data (solid line for spaxels, dashed line for individual H II regions), assuming the mean and standard-deviation of the distribution of each analysed parameter and sampled with the same bins.

Table 1. Oxygen abundance gradient slopes derived using different calibrators and distance normalisations.

\begin{tabular}{ccccccc}
\hline \hline Calibrator & & \multicolumn{2}{c}{$\alpha_{\mathrm{O} / \mathrm{H}}-$ spaxels } & \multicolumn{3}{c}{$\alpha_{\mathrm{O} / \mathrm{H}-\mathrm{H} \text { II regions }}$} \\
& {$\left[\mathrm{dex} / r_{\mathrm{e}}\right]$} & {$\left[\mathrm{dex} / r_{25}\right]$} & {$[\mathrm{dex} / \mathrm{kpc}]$} & {$\left[\mathrm{dex} / r_{\mathrm{e}}\right]$} & {$\left[\mathrm{dex} / r_{25}\right]$} & {$[\mathrm{dex} / \mathrm{kpc}]$} \\
\hline O3N2 [M13] & $-0.07 \pm 0.05$ & $-0.08 \pm 0.06$ & $-0.009 \pm 0.008$ & $-0.05 \pm 0.06$ & $-0.06 \pm 0.06$ & $-0.008 \pm 0.010$ \\
O3N2 [PP04] & $-0.11 \pm 0.07$ & $-0.12 \pm 0.09$ & $-0.014 \pm 0.012$ & $-0.08 \pm 0.09$ & $-0.09 \pm 0.09$ & $-0.011 \pm 0.014$ \\
ONS [P10] & $-0.06 \pm 0.06$ & $-0.08 \pm 0.07$ & $-0.008 \pm 0.009$ & $-0.06 \pm 0.09$ & $-0.08 \pm 0.11$ & $-0.008 \pm 0.012$ \\
pyqz [D13] & $-0.14 \pm 0.09$ & $-0.15 \pm 0.10$ & $-0.019 \pm 0.014$ & $-0.11 \pm 0.09$ & $-0.13 \pm 0.11$ & $-0.015 \pm 0.015$ \\
\hline
\end{tabular}

$\left.S_{2}=[\mathrm{S} \mathrm{II}](\lambda 6717+\lambda 6731)\right)$ as temperature and metallicity indexes and is valid over the whole range of explored metallicities. The derived relations to determine the oxygen abundances using this calibration are given by P10. Finally, the D13 calibration is based on a grid of photoionisation models covering a wide range of abundance and ionisation parameters typical of $\mathrm{H}$ II regions in galaxies. This calibration can be used through a Python module implemented by the authors, known as pyqz, which is publicly available ${ }^{4}$.

Table 1 shows a comparison among the oxygen abundance slopes derived using the different calibrators and the different normalisations for the radial distance described before. In this table we present the values using both the spaxels and the individual $\mathrm{H}$ II regions.

We also illustrate this comparison in Fig. 6. The left panel represents the distribution of oxygen abundances derived using the M13 calibration for the O3N2 index vs. the P10 calibration for the ONS index. In the right panel we show the same distribution of the M13 calibration, but this time vs. the D13 calibration based on the pyqz code. Both panels show a tight correlation between the compared calibrators. This allows us to conclude that our qualitative results are not contingent upon the choice of the used calibrator, although the actual measured values for the abundance gradients may change. Similar conclusions were stated by Ho et al. (2015) for a different sample of galaxies.

For the sake of clarity, below we only show the results of this article based on the use of the M13 calibrator. However, we have

\footnotetext{
4 http://dx.doi.org/10.4225/13/516366F6F24ED
}
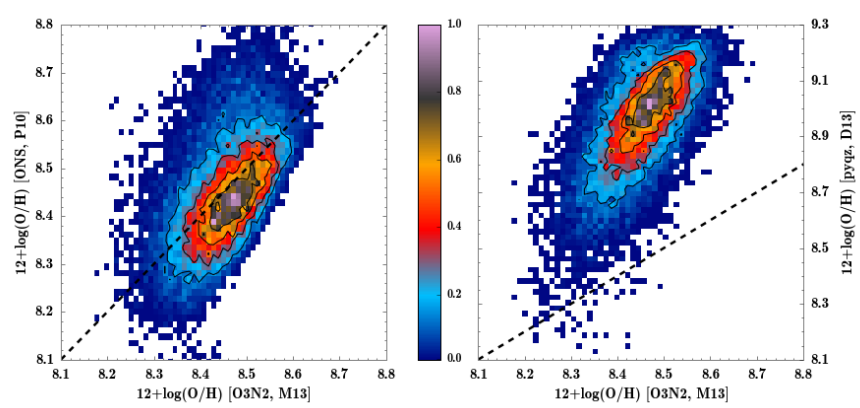

Fig. 6. Comparison of the oxygen abundance distribution derived using the calibration proposed by M13 for the O3N2 index with the distribution derived using the P10 calibration for the ONS index (left panel) and the calibration based on pyqz code (D13, right panel). The black contours show the density distribution of the SF spaxels, the outermost one including $80 \%$ of the total number of spaxels and decreasing $20 \%$ in each consecutive contour. The black dashed lines indicate the 1:1 relation between the represented calibrators.

reproduced the analysis using all the proposed calibrators, with no significant differences between the obtained results.

\subsection{Abundance gradients by galaxy types}

After we derived the oxygen abundance gradient for all galaxies, we analysed whether there was a dependence of the slope on different properties of the galaxies. We focused this analysis on 

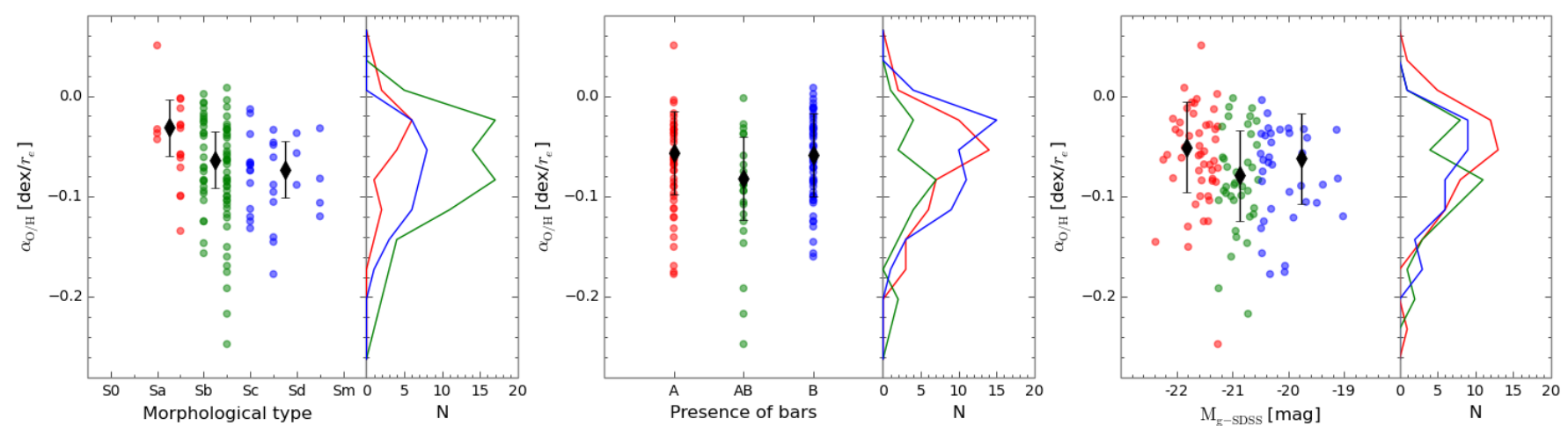

Fig. 7. Distribution of the abundance slopes as a function of the morphological type of the galaxies (left panel), depending on the presence or absence of bars (middle panel) and as a function of the $g$-band absolute magnitude of the galaxies (right panel). We also show the histograms for each distribution, where $N$ is the number of galaxies and colours indicate the different classification types: (i) early spirals, Sa-Sab (red); intermediate spirals, Sb-Sbc (green); late spirals, Sc-Sm (blue) for the left panel. (ii) Clearly unbarred galaxies (red); clearly barred (blue); an intermediate stage (green) for the middle panel. (iii) Luminous galaxies, $M_{g \text {-SDSs }}<-21.25 \mathrm{mag}$ (red); intermediate galaxies, $-21.25<$ $M_{g \text {-SDSs }}<-20.5 \mathrm{mag}$ (green); faint galaxies, $M_{g \text {-SDSs }}>-20.5 \mathrm{mag}$ (blue) for the right panel. Black diamonds represent the median values for the distributions, together with the standard deviation shown as error bars.

three properties: the differences in the morphological type, the effect of the bars, and the luminosity of the galaxies. We showed in Sect. 4.1 that the oxygen abundance distribution obtained for both the spaxels and H II regions are equivalent and accordingly lead to the same results, therefore we carried this analysis out for the spaxel-wise information alone.

Figure 7 shows the slope distribution as a function of the morphological type of the galaxy (left panel), the presence or absence of bars (middle panel), and the $g$-band absolute magnitude of the galaxies (right panel). We also show the histograms for each distribution, where $N$ is the number of galaxies. The limits used in the classification based on the luminosity of the galaxies were chosen to ensure a similar number of elements in each bin (i.e. comparable from a statistical point of view). We tried to follow a similar criterion in the separation by morphological types, but the deficiency of Sa-Sab galaxies and the criterion of not considering $\mathrm{Sb}$ as early type prevented us from obtaining a comparable number of objects in each bin.

The slope distribution by morphological types seems to vary in a way that the earlier spirals present flatter gradients than the later type ones. The median values for the distributions together with the standard deviations are

$\mathrm{Sa}-\mathrm{Sab}: \alpha_{\mathrm{O} / \mathrm{H}}=-0.04 \mathrm{dex} / r_{\mathrm{e}}$ and $\sigma=0.04 \mathrm{dex} / r_{\mathrm{e}}\left(n_{g a l}=17\right)$

$\mathrm{Sb}-\mathrm{Sbc}: \alpha_{\mathrm{O} / \mathrm{H}}=-0.07 \mathrm{dex} / r_{\mathrm{e}}$ and $\sigma=0.05 \mathrm{dex} / r_{\mathrm{e}}\left(n_{\text {gal }}=74\right)$

$\mathrm{Sc}-\mathrm{Sm}: \alpha_{\mathrm{O} / \mathrm{H}}=-0.07 \mathrm{dex} / r_{\mathrm{e}}$ and $\sigma=0.04 \mathrm{dex} / r_{\mathrm{e}}\left(n_{\text {gal }}=31\right)$.

We performed a two-sample Kolmogorov-Smirnov test (KStest) to check if the differences found between the distributions are significant. The significance level of the KS-test is 5\%, meaning that values below this limit come from different distributions. We derived a $P$-value of $7 \%$ for the distributions with the largest differences (between early and late spirals), a $P$-value of $10 \%$ for the test comparing the early and intermediate types, and a $P$-value of $92 \%$ between intermediate and late ones. We also performed an Anderson-Darling test (AD-test), which is more suitable when the samples comprise only few objects, with a resulting $P$-value of $8 \%$ for the early-late comparison, a $P$ value of $16 \%$ for the test comparing the early and intermediate type, and a $P$-value of $73 \%$ in the intermediate-late case. This clearly shows that the observed differences are negligible.

For the distribution of slopes depending on the presence or absence of bars we defined three different groups: galaxies with no bar (A), galaxies that may have a bar, but where the bar is not clearly visible (AB), and clearly barred galaxies (B). The results are as follows:

$$
\begin{array}{ll}
\mathrm{A}: & \alpha_{\mathrm{O} / \mathrm{H}}=-0.06 \mathrm{dex} / r_{\mathrm{e}} \text { and } \sigma=0.05 \mathrm{dex} / r_{\mathrm{e}}\left(n_{\text {gal }}=46\right) \\
\mathrm{AB}: & \alpha_{\mathrm{O} / \mathrm{H}}=-0.09 \mathrm{dex} / r_{\mathrm{e}} \text { and } \sigma=0.06 \mathrm{dex} / r_{\mathrm{e}}\left(n_{\text {gal }}=23\right) \\
\mathrm{B}: & \alpha_{\mathrm{O} / \mathrm{H}}=-0.06 \mathrm{dex} / r_{\mathrm{e}} \text { and } \sigma=0.04 \mathrm{dex} / r_{\mathrm{e}}\left(n_{\text {gal }}=53\right) .
\end{array}
$$

We found negligible differences for these distributions. This was confirmed by the KS test, which gives a $P$-value of $52 \%$ for the comparison of the A-AB distributions, a $P$-value of $50 \%$ for the A-B distributions, and a $P$-value of $10 \%$ for the AB-B distributions (from the AD-tests we obtain $P$-values of $39 \%, 27 \%$ and $5 \%$ for each bin, respectively).

Finally, to analyse the distribution of slopes depending on the luminosity of the galaxies, we divided the sample again into three groups: luminous (L, $M_{g \text {-SDSS }}<-21.25 \mathrm{mag}$ ), intermediate (I, $-21.25<M_{g \text {-SDSS }}<-20.5 \mathrm{mag}$ ), and faint $(\mathrm{F}$, $M_{g \text {-SDSS }}>-20.5 \mathrm{mag}$ ) galaxies. We obtained these results:

$$
\begin{array}{ll}
\mathrm{L}: & \alpha_{\mathrm{O} / \mathrm{H}}=-0.06 \mathrm{dex} / r_{\mathrm{e}} \text { and } \sigma=0.05 \mathrm{dex} / r_{\mathrm{e}}\left(n_{g a l}=49\right) \\
\mathrm{I}: & \alpha_{\mathrm{O} / \mathrm{H}}=-0.09 \mathrm{dex} / r_{\mathrm{e}} \text { and } \sigma=0.05 \mathrm{dex} / r_{\mathrm{e}}\left(n_{g a l}=37\right) \\
\mathrm{F}: & \alpha_{\mathrm{O} / \mathrm{H}}=-0.07 \mathrm{dex} / r_{\mathrm{e}} \text { and } \sigma=0.05 \mathrm{dex} / r_{\mathrm{e}}\left(n_{g a l}=36\right) .
\end{array}
$$

The statistical tests yield a $P$-value of $13 \%$ for the KS-test in the case with the largest differences, that is, when comparing luminous and intermediate galaxies. The remaining KS-tests return a $P$-value of $67 \%$ for the comparison between luminous and faint galaxies and a $P$-value of $72 \%$ when analysing the intermediate and faint distributions (a $P$-value of 19\%, 50\%, and 52\%, respectively, for the $\mathrm{AD}$-tests).

Similar results for all these separations are found when using any scale-length normalisation for the radial distance instead of the disc effective radius, either the $r_{25}$ radius or the physical scale of the galaxy (i.e. the radial distance in $\mathrm{kpc}$ ).

\subsection{Common abundance gradient}

The fact that the distribution of the oxygen abundance gradients for all the galaxies in the sample is well fitted by a Gaussian function suggests the existence of a characteristic value for the slope, independent of other properties of the galaxies, as the 

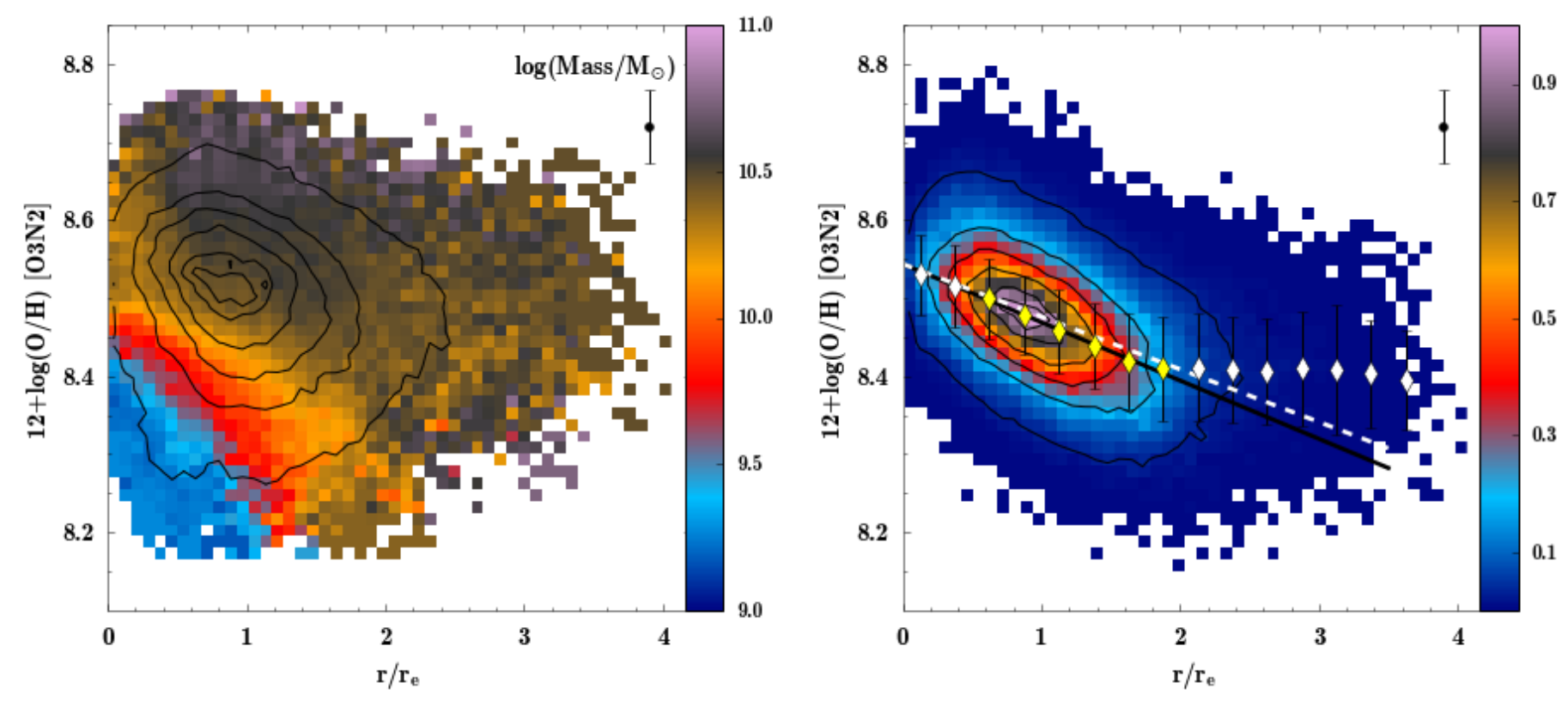

Fig. 8. Left: radial distribution of the oxygen abundance derived using the $\mathrm{O} 3 \mathrm{~N} 2$ indicator and the calibration proposed by M13 for all the galaxies in our sample. The black contours show the density distribution of the star-forming spaxels, the outermost one including $95 \%$ of the total number of spaxels and decreasing $20 \%$ in each consecutive contour. The colour bar displays the average stellar mass of each galaxy (in log scale) corresponding to each abundance and radial distance. The average error of the derived oxygen abundances is indicated as an error bar located at the top right side of the panel. For clarity, only the oxygen abundance values with a contribution of at least $1 \%$ of the total number of spaxels are plotted. Right: radial density distribution of the oxygen abundance after rescaling the oxygen abundances of each galaxy following the $\mathcal{M}-\mathcal{Z}$ relation derived in Sánchez et al. (2013). As in the left panel, the outermost contour encircles 95\% of the total number of spaxels, decreasing $20 \%$ in each consecutive contour. The diamonds represent the mean oxygen abundance values, with the error bars indicating the corresponding standard deviations, for bins of $0.25 r_{\mathrm{e}}$. The solid-black line represents the error-weighted linear fit derived for those mean values within the range between 0.5 and $2.0 r_{\mathrm{e}}$ (yellow diamonds), and the dashed white line represents the linear relation corresponding to the characteristic values of the zero-points and slopes derived in Sect. 4.1 for the individual galaxies assuming a Gaussian distribution for both parameters.

morphological type or the luminosity. This is true when normalising the radial galactocentric distances to the disc effective radius and limiting the fitted interval between 0.5 and $2.0 r_{\mathrm{e}}$. Below this range $\left(r / r_{\mathrm{e}}<0.5\right)$, a nearly flat distribution or even a drop towards the centre is found for some galaxies of the sample. At larger galactocentric distances $\left(r / r_{\mathrm{e}}>2.0\right)$, a flattening can be observed in the abundance gradient of most of the galaxies.

It is easier to illustrate this result if we represent the radial distribution of the oxygen abundance for all the galaxies in the same figure. This is shown in the left panel of Fig. 8. The black contours represent the density distribution of the star-forming spaxels. Although the radial gradient can be discerned through the contour plot, the wide range of abundances blurs the result. To clarify the origin of this widening, we colour-code each represented abundance according to the integrated stellar mass of the host galaxy in log scale. For clarity, only the oxygen abundance values with a contribution of at least $1 \%$ of the total number of spaxels are plotted. Adopting this scheme, it is evident that the abundances present a common radial gradient, but with an offset depending on the mass, as expected from the $\mathcal{M}-\mathcal{Z}$ relation (and as discussed in Sect. 4.1). To remove this dependence on the mass from the map, we rescaled the oxygen abundances of each galaxy following the analytical form of the $\mathcal{M}-\mathcal{Z}$ relation (Tremonti et al. 2004) derived by Sánchez et al. (2013), applying an offset according to the integrated stellar mass of the galaxy and normalising to the average value for the whole sample of $\sim 8.5$. The outcome is shown in the right panel of Fig. 8, where the common abundance gradient presented by all galaxies in the sample can be clearly seen. The abundance distribution is represented again as a density map, both with a colour-code image (normalised to one) and a contour map. The diamonds represent the mean oxygen abundance values, with the error bars indicating the corresponding standard deviations, for bins of 0.25 $r_{\mathrm{e}}$. An error-weighted linear regression (solid black line) to the mean values restricted to the spatial range between 0.5 and 2.0 $r_{\mathrm{e}}$ (yellow diamonds) derives a slope of $\alpha_{\mathrm{O} / \mathrm{H}}=-0.075 \mathrm{dex} / r_{\mathrm{e}}$ and $\sigma=0.016 \mathrm{dex} / r_{\mathrm{e}}$, totally compatible with the characteristic slope of $\alpha_{\mathrm{O} / \mathrm{H}}=-0.07 \pm 0.05 \mathrm{dex} / r_{\mathrm{e}}$ derived in Sect. 4.1 for the individual galaxies assuming a Gaussian distribution (dashedwhite line).

This characteristic slope is independent of the integrated stellar mass of the galaxies. Figure 9 shows the mean radial profiles of the oxygen abundance for our sample of galaxies split in four mass bins with a similar number of elements $\left(\log \left(M / M_{\odot}\right) \leq\right.$ 10.2 , blue diamonds; $10.2<\log \left(M / M_{\odot}\right) \leq 10.5$, red squares; $10.5<\log \left(M / M_{\odot}\right) \leq 10.75$, yellow dots; $\log \left(M / M_{\odot}\right) \geq 10.75$, purple triangles). The derived gradients (computed between 0.5 and $2.0 r_{\mathrm{e}}$ ) of those four profiles are consistent with the characteristic slope displayed by the entire sample.

All these gradients were derived up to two disc effective radii. As we have already mentioned, previous studies have found that galaxies present a flattening in the abundance when covering regions beyond $r_{25}$, observed both in the stellar populations (e.g. Yong et al. 2006; Carraro et al. 2007; Vlajić et al. 2009, 2011) and in the gas (e.g. Martin \& Roy 1995; Vilchez \& Esteban 1996; Roy \& Walsh 1997; van Zee et al. 1998; Bresolin et al. 2009, 2012; Rosales-Ortega et al. 2011; Marino et al. 2012, 2016; López-Sánchez et al. 2015). The right panel of Fig. 8 shows the appearance of the mentioned flattening in the oxygen abundance distribution beyond $\sim$ two effective radii, with around 


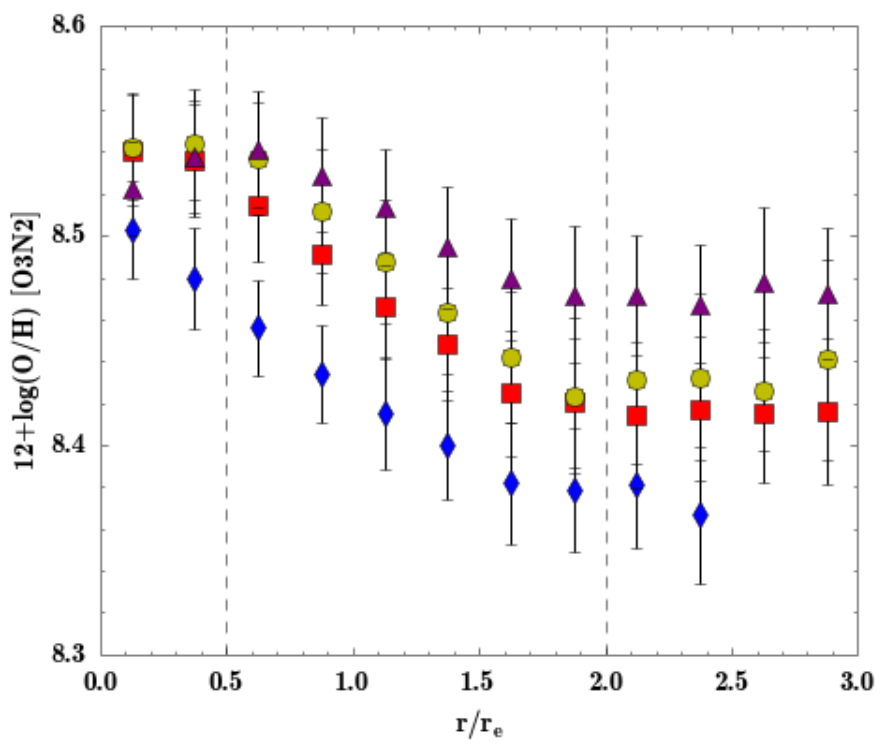

Fig. 9. Mean oxygen abundance radial profiles derived considering galaxies in four different bins according to their integrated stellar mass. The limits of the bins were chosen to ensure a similar number of elements in each bin: $\log \left(M / M_{\odot}\right) \leq 10.2$, blue diamonds; $10.2<\log \left(M / M_{\odot}\right) \leq 10.5$, red squares; $10.5<\log \left(M / M_{\odot}\right) \leq 10.75$, yellow dots; $\log \left(M / M_{\odot}\right) \geq 10.75$, purple triangles. The symbols represent the mean oxygen abundance values, with the error bars indicating the corresponding standard deviations, for bins of $0.25 r_{\mathrm{e}}$. Dashed vertical lines delimit the three different behaviours in the oxygen abundance profiles (i.e. drop in the inner parts, common gradient between 0.5 and $2.0 r_{\mathrm{e}}$, and the flattening in the outer parts).

8230 spaxels at these outer regions. By inspecting the galaxies individually, we detected this flattening in 57 of them, corresponding to $82 \%$ of the galaxies with reliable oxygen abundance values at these large galactocentric distances. The onset of the flattening is always located around $2 r_{\mathrm{e}}$ even if we separate the sample in different bins according to the integrated stellar mass (see Fig. 9). In the same figure we show that the abundance value of the flattening depends on the mass of the galaxies because of the mentioned $\mathcal{M}-\mathcal{Z}$ relation.

In addition to this flattening in the outer parts, we found that $27(22 \%)$ galaxies of the sample display some anomalies in their oxygen abundance profiles in the inner parts, namely a nearly flat distribution (8 galaxies, $6.5 \%$ ) or even a drop towards the centre (15 galaxies, $15.5 \%$ ). This feature has also been found in previous works (e.g. Belley \& Roy 1992; Rosales-Ortega et al. 2011; Sánchez et al. 2012b, 2014). The presence of this feature is not visible when representing the radial distribution of the oxygen abundance for all the galaxies in our sample (Fig. 8), but it does appear when separating the sample into different stellar mass bins (Fig. 9), only in the case of the more massive galaxies. While the lowest stellar mass galaxies do not display any sign of this feature, this drop is progressively more evident with increasing galaxy mass.

\section{Discussion and conclusions}

At the beginning of this article we have pointed out the importance of studying the chemical abundance to understand the evolution of galaxies. In particular, observational studies of the spatial distribution of chemical abundances and its cosmological evolution allow us to set strong constraints for chemical evolution models that try to explain the formation processes of disc galaxies (Koeppen 1994; Edmunds \& Greenhow 1995; Tsujimoto et al. 1995; Mollá et al. 1997; Prantzos \& Boissier 2000; Chiappini et al. 2001; Mollá \& Díaz 2005; Fu et al. 2009; Pilkington et al. 2012; Mott et al. 2013).

The existence of a radial decrease in the chemical abundances of nearby spiral galaxies has been well established by observations for decades (Searle 1971; Comte 1975; Smith 1975; Peimbert 1979; Shaver et al. 1983). Since then, this gradient has been confirmed by other works, restricted to individual galaxies or to limited samples of galaxies (Martin \& Roy 1992; Kennicutt et al. 2003; Rosales-Ortega et al. 2011; Bresolin et al. 2012; Marino et al. 2012; Patterson et al. 2012). With the advent of IFS surveys like CALIFA, abundance studies using larger samples of H II regions have become feasible (Sánchez et al. 2012b, 2014), with the same results as in previous studies.

In this work we went a step further and analysed for the first time the oxygen abundance distribution for a large sample of galaxies spaxel by spaxel, taking advantage of the full $2 \mathrm{D}$ information provided by the CALIFA survey, which improves the statistics. This final sample provides more than 185000 oxygen abundance values and more than $7100 \mathrm{HII}$ regions with which to compare our results. The analysis using the H II regions was previously performed by $\mathrm{S} 14$ with a different sample of galaxies that were also extracted from the CALIFA mother sample. With spaxel-by-spaxel analysis, we here took approximately four times more line emitting spaxels into account that are associated with star formation regions than in the classical procedure of detecting H II regions. Our results are therefore mainly based on an independent set of measurements, reinforcing and expanding on the S14 results. A more complete 2D study of the oxygen abundance distribution, analysing possible azimuthal variations, will be the topic of a forthcoming work. In this paper, we focused on the radial distribution of the oxygen abundances.

\subsection{Common abundance gradient}

Our results confirmed the radially decreasing abundance that was described above and the existence of a characteristic gradient in the oxygen abundance within 0.5-2.0 disc effective radii, independent of other galaxy properties, which is similar to the result reported previously by Sánchez et al. (2012b, 2014).

The distribution of the derived oxygen abundance gradients is fully compatible with being Gaussian, presenting a characteristic value for the slope of $-0.07 \mathrm{dex} / r_{\mathrm{e}}$ and a standard deviation of $0.05 \mathrm{dex} / r_{\mathrm{e}}$. We estimated that at least a $50 \%$ of the dispersion could be justified by the nominal errors in the derivation of the slopes.

To assess a possible dependence of the gradient distribution on different properties of the galaxies, we studied the abundance gradient distribution of different subsamples according to the morphological type, the effect of bars, and the luminosity of the galaxies. Based on this analysis, we did not find statistically significant differences (in terms of KS-tests) between them.

The results of this analysis contradict some previous studies, which did find a relation between the slope in the gas abundance gradient and some properties of the galaxies, such as the morphology, the mass, or the presence of bars. The dependence of the slope on the morphology of the galaxies is still an open question. Early studies found a correlation between the abundance slope and the morphological type of galaxies, with later types showing steeper gradients (Vila-Costas \& Edmunds 1992; Oey \& Kennicutt 1993). Other studies, however, suggested gradients independent of galaxy type (Diaz 1989; Zaritsky et al. 1994), 
when normalising to a physical scale of the disc (e.g. the $r_{25}$ radius or the disc scale-length $r_{\mathrm{d}}$ ). The contradictory results might be due to the small and heterogeneous samples and inconsistent methods of measuring metallicity gradients.

For the effect of bars the conclusions likewise disagree. It is well known that roughly $30-40 \%$ of the spiral galaxies have a strong bar in optical wavelengths, $60 \%$ if we take into account weaker bars (e.g. Sellwood \& Wilkinson 1993; Marinova \& Jogee 2007; Sheth et al. 2008; Aguerri et al. 2009; Masters et al. 2011). Gas kinematic data show the presence of strong non-circular motions in bars (Huntley 1978; Zurita et al. 2004; Holmes et al. 2015), indicating that the bar constitutes a major non-axisymmetric component of the galaxy mass distribution (Sellwood \& Wilkinson 1993). Bars have been proposed as a key mechanism in the dynamical evolution of disc galaxies. For instance, they are able to contribute to the redistribution of matter in the galaxy by exchanging angular momentum with the disc, inducing gas flows (Athanassoula 1992; Friedli 1998). This radial movement can produce a mixing and homogenisation of the gas, changing the abundance profiles in the disc and causing a flattening in the gas abundance gradients (Friedli et al. 1994; Friedli \& Benz 1995; Portinari \& Chiosi 2000; Cavichia et al. 2014).

Different studies have observed this flattening in the gas abundance gradient of barred galaxies and found a correlation between the abundance slope and the presence of a bar (Vila-Costas \& Edmunds 1992; Martin \& Roy 1994; Zaritsky et al. 1994), independently of the adopted normalisation radius. However, recent works on either stellar or gas-phase metallicity have not found evidence of such a correlation between them (Sánchez et al. 2012b, 2014; Sánchez-Blázquez et al. 2014; Cheung et al. 2015). Our results support this absence of a correlation between oxygen gradient and the presence of bars. However, stellar radial migration due to bars may enlarge the effective radius of the disc (Debattista et al. 2006), which could compensate for the effect on the oxygen abundance values and produce the same gradient as in absence of bars. We have checked this possibility and confirm that there is no such effect on the disc effective radius, as can be inferred from the bottom right panel of Fig. 1. This fact, together with the absence of correlation between oxygen gradient and the presence of bar, suggests that bars alone may not have such a strong influence on the chemical evolution of disc galaxies as predicted by simulations.

We have tried to describe the overall effect of the presence of a bar on the abundance gradients (among other proporties of galaxies), obtaining that bars alone may not affect the chemical evolution of disc galaxies so strongly. However, the relative size of the bar with respect to the disc might play an important role on the derived gradients. To assess this possibility, we need to properly measure the size of the bars, a task that is hampered by the sometimes elusive nature of these structures. This parameter will be determined as a result of the photometric analysis currently carried out by the CALIFA collaboration (Méndez-Abreu et al., in prep.). Thus, we leave the analysis of this possible effect for a future work.

Finally, no trends with the luminosity or mass of the galaxies have been found in previous studies when normalising to a physical scale of the disc (Zaritsky et al. 1994; Garnett et al. 1997; Ho et al. 2015); this agrees with our results. We note that several studies that found correlations with some properties of the galaxies measured the abundance gradients in absolute scale (i.e. $\mathrm{kpc}$ ). When normalising the galactocentric distances to scale lengths such $r_{25}, r_{\mathrm{d}}$ or $r_{\mathrm{e}}$, the correlations sometimes disappear. This can partially be understood as a size effect. If galaxies with steeper metallicity gradients measured in dex $\mathrm{kpc}^{-1}$ are smaller in their physical sizes ( $\mathrm{small} r_{25}$ ), then the steep dex $\mathrm{kpc}^{-1}$ metallicity gradients would be compensated for when the galaxy sizes are taken into account. Sánchez et al. (2012b, 2013) stated the importance of defining the gradient normalised to the disc effective radius, since this parameter presents a clear correlation with other properties of the galaxies, such as the absolute magnitude, the mass, or the morphological type.

Both the fact that the distribution of the derived oxygen abundance gradients is compatible with a Gaussian distribution and this lack of correlations between the slope values and the analysed properties of galaxies support the existence of a characteristic value for the slope that is common to all type of spiral galaxies (interacting galaxies were not considered in the study and are not included in this statement).

Several works have detected the mentioned gradient in the MW (e.g. Shaver et al. 1983; Deharveng et al. 2000; Pilyugin et al. 2003; Esteban et al. 2005; Quireza et al. 2006; Rudolph et al. 2006; Balser et al. 2011), deriving values for the slope between -0.04 and $-0.06 \mathrm{dex} / \mathrm{kpc}$. If we express the gradient in $\mathrm{dex} / r_{\mathrm{e}}$ to easily compare with our results, and considering a value of the disc effective radius for our Galaxy of $r_{\mathrm{e}}=3.6 \mathrm{kpc}$ (Bovy \& Rix 2013), we obtain a slope between -0.14 and $-0.22 \mathrm{dex} / r_{\mathrm{e}}$, which is slightly larger than the mean value of our slope distribution ( $-0.07 \mathrm{dex} / r_{\mathrm{e}}$, see Table 1$)$. However, deriving the abundance gradient for the MW is not straightforward and presents several obstacles: (i) the measurements are affected by dust and it is difficult to determine distances, which is necessary to derive radial abundance gradients; (ii) the use of different abundance diagnostics such as optical and IR collisionally excited lines (CELs), optical and radio recombination lines (RRL) or thermal continuum emission can yield differences in the results (Rudolph et al. 2006); (iii) azimuthal abundance variations have been reported in the MW (Pedicelli et al. 2009; Balser et al. 2011) that would complicate any analysis of the radial gradients; and finally (iv) the value of the disc scale-length, and therefore the disc effective radius, is still controversial, with large discrepancies among different results (typical values are in the range 2-4 kpc, e.g. Sackett 1997; Hammer et al. 2007; van der Kruit \& Freeman 2011; Bovy \& Rix 2013). All these factors may contribute to an incorrect estimate of the oxygen abundance gradient in our Galaxy, which would explain the differences found in this value between the MW and external galaxies. Another aspect to take into account is the different radial range considered in the derivation of the gradient. While in our work the gradients were computed between 0.5 and $2.0 r_{\mathrm{e}}$ (see Sect. 3.5), it is obtained within a typical range between 5 and $15 \mathrm{kpc}$ for the MW, which roughly corresponds to 1.4 and $4.2 r_{\mathrm{e}}$ (using again a value of $3.6 \mathrm{kpc}$ for the $r_{\mathrm{e}}$, Bovy \& Rix 2013). This fact may also contribute to the differences in the gradient values.

The origin of this negative abundance gradient goes back to the inside-out scenario for the formation of disc galaxies (described in Sect. 1), where the inner parts form first, followed by the formation of the outer regions. Further evidence in the CALIFA survey comes from the analysis of the SP, either from the study of the SFH (Pérez et al. 2013) or from the study of the radial age gradient (González Delgado et al. 2014, 2015; RuizLara et al. 2016). This scenario is understood as a result of the increased timescales of the gas infall with radius and the consequent radial dependence of the SFR. This radial decrease in the chemical abundance has been well established since a long time (Searle 1971). However, the statement that this gradient presents a characteristic slope independent of many properties of the galaxies was only recently expressed (Sánchez et al. 2012b), 
which imposes stronger restrictions on our current understanding of disc galaxy growth. The existence of a common gradient in the abundances indicates that the chemical evolution of these disc-dominated galaxies is tightly correlated with the mass growth.

\subsection{Flattening of the abundance gradient in the outer regions}

Several studies analysing the gas content on galaxies have recently found that beyond the isophotal radii $r_{25}$, the metallicity radial distribution flattens to a constant value independent of the galactocentric distance, in contrast to the negative abundance gradient present at smaller distances (e.g. Martin \& Roy 1995; Vilchez \& Esteban 1996; Roy \& Walsh 1997; van Zee et al. 1998; Bresolin et al. 2009, 2012; Werk et al. 2010, 2011; Rosales-Ortega et al. 2011; Marino et al. 2012; Sánchez et al. 2012b; López-Sánchez et al. 2015). This change in the abundance distribution has not only been detected in the gas, but also in the stellar metallicity (e.g. Yong et al. 2006; Carraro et al. 2007; Vlajić et al. 2009, 2011). However, all these works were based on individual galaxies or a very limited sample of objects. S14 represents the first unambiguous detection of such a flattening in a statistically significant large sample of galaxies. This is the first work confirming the flattening by using spaxel-byspaxel information, increasing the number statistics and improving the spatial coverage across the discs.

Average radial distributions of oxygen abundances similar to the distribution shown in Fig. 8 were created for the same subsamples analysed in Sect. 4.2 (i.e. according to the morphological type, presence or absence of bars and luminosity). No significant differences were found between them, which means that the flattening at the outer regions seems to be a universal property of spiral galaxies. It is also important to note that although Fig. 8 was created using the M13 indicator based on the $\mathrm{O} 3 \mathrm{~N} 2$ index, the flattening is independent of the adopted calibrator; it is present when performing the analysis with all the explored calibrators (as indicated before in Sect. 4.1.1).

The nature of this flattening is still under debate. Because of the extreme conditions of the outermost parts of galaxies (very low gas densities and long dynamical timescales), these regions play a key role in studying the mechanisms involved in their evolution and, therefore, the existence of this flattening can be of great importance in constraining chemical evolution models.

The flattening, although observed in the more metal poor regions of the galaxies, displays a relatively high abundance value, which ranges between 8.4 and 8.6 (left panel of Fig. 8). An estimate of the time necessary to enrich the ISM of the outer parts of discs to these abundance levels, assuming a constant SFR equal to the present observed value, is higher than $10 \mathrm{Gyr}$ (see Bresolin et al. 2012, for details of the calculation). According to cosmological hydrodynamical simulations, in the inside-out growth scenario the outer regions of galaxy discs are formed during the last 4-6 Gyr (Scannapieco et al. 2008, 2009). Therefore, if star formation has proceeded at the same rate as observed today, the enrichment of the ISM caused by stellar evolution in these outer parts cannot produce these observed high gas abundance values during the lifetime of these regions.

Different mechanisms have been proposed to explain this enrichment in the outer parts of discs in non-interacting galaxies. One of them is a metal-mixing scenario induced by large-scale processes of angular momentum transport, such as radial gas flows (Lacey \& Fall 1985; Goetz \& Koeppen 1992; Portinari \& Chiosi 2000; Ferguson \& Clarke 2001; Schönrich \& Binney 2009; Bilitewski \& Schönrich 2012; Spitoni et al. 2013, among others), resonance with transient spiral structure (Sellwood \& Binney 2002; Minchev et al. 2012; Roškar et al. 2012; Daniel \& Wyse 2015), or the overlap of spiral and bar resonances (Minchev \& Famaey 2010; Minchev et al. 2011). In the chemical evolution model developed for the MW by Cavichia et al. (2014), the presence of the bar induces radial gas flows in the disc whose net effect is to produce this flattening of the oxygen gradient at the outer disc. However, as pointed out by the authors, the differences are small compared with the model without the bar and probably cannot be distinguished by the observations. Minor mergers and perturbations caused by orbiting satellite galaxies are also suggested to increase the metal content in the external regions (Quillen et al. 2009; Qu et al. 2011; Bird et al. 2012; López-Sánchez et al. 2015). A slow radial dependence of the star formation efficiency (SFE) at large galactocentric distances is another possible explanation to the flattening (Bresolin et al. 2012; Esteban et al. 2013). An alternative interpretation arises from recent cosmological simulations that propose a balance between outflows and inflows (through 'wind recycling' accretion) with the intergalactic medium (IGM) as a mechanism governing the gaseous and metal content of galaxies (Oppenheimer \& Davé 2008; Oppenheimer et al. 2010; Davé et al. 2011, 2012). Finally, it may well be that a fraction of the metals are recycled in the halo of the galaxy and do not escape, being mixed with the incoming gas, as mentioned by Veilleux et al. (2005), and producing the observed flattening.

All these mechanisms are not mutually exclusive, and a possible balance between them could be responsible for the actual chemical evolution of extended discs and the flat abundance of the outermost parts. Moreover, the dominant mechanism could be different for structurally different galaxies. However, our current results do not allow disctinguishing between the different mechanisms; additional information is needed to assess this question.

\subsection{Abundance decrease in the inner regions}

As a deviation from the monotonic behaviour of the oxygen abundances increasing towards the centre, several studies have found that galaxies sometimes present a nearly flat distribution of abundances or even a drop in the inner regions, at $r / r_{\mathrm{e}}<0.3-0.5$ (Belley \& Roy 1992; Rosales-Ortega et al. 2011; Sánchez et al. 2012b, 2014). This feature has also been detected in simulations (Mollá \& Díaz 2005; Cavichia et al. 2014).

For our Galaxy, the studies on gas abundance have not been able to properly trace the oxygen abundance at these innermost parts of the disc and therefore were not able to find evidence of this behaviour. However, a chemical evolution model developed by Cavichia et al. (2014) has detected this "drop" (or flattening) in the gas abundances of the inner regions as caused by the presence of a bar. According to this model, the presence of the bar induces radial flows that increase the SFR at the corotation radius, which also produces an increase in the oxygen abundance. This results in an apparent decrease or flattening of the abundance in the central regions. A recent study focused on stellar populations (Hayden et al. 2014) has detected a flattening in the metallicity of the inner regions of the Galaxy, more significant in the low$[\alpha / \mathrm{M}]$ ( $\alpha$ element abundances) stars. They also explained this feature by the existence of a central bar that produces a mixing of stars in these inner regions.

The analysis presented here can help us to confirm this scenario where the inner drop is caused by the influence of a bar. If the bar were the dominant effect that produces this decrease at inner regions, then it would be expected to detect it more 
frequently in barred galaxies. However, our analysis yields that only $30 \%$ of galaxies in the sample showing the drop are barred (52\% if we also consider the galaxies that are supposed to have a bar but where it is not clearly visible).

Another explanation proposed by observational studies on gas abundance for external galaxies, like NGC 628 (Sánchez et al. 2011) and later using CALIFA data in a large sample of galaxies (S14), is that this inner decrease was associated with the presence of a circumnuclear star-forming ring of ionised gas related to the inner Limbland resonance.

To explore the possibility that the abundance drop is due to the presence of a star-forming ring, a visual inspection of the $\mathrm{H} \alpha$ intensity maps for the galaxies with signs of this drop was carried out. This analysis showed that $48 \%$ of the galaxies present evidence of a star-forming ring that is spatially located at these inner galactocentric distances $\left(r / r_{\mathrm{e}} \sim 0.3-0.5\right)$. However, this scenario must be confirmed by a more robust and detailed analysis of the stellar and gas kinematics.

Despite these two possible explanations (influence of a bar or a circumnuclear star-forming ring), the physical origin of this drop is not still well established. On the other hand, if the origin of this drop were due to radial motions of the gas, it would also have an impact on the overall distribution of abundances at larger radii. To explore this possible effect, we derived the mean value for the slope of the abundance gradients only for the galaxies presenting this feature, obtaining a mean $\alpha_{\mathrm{O} / \mathrm{H}}=-0.10 \mathrm{dex} / r_{\mathrm{e}}$ and $\sigma=0.04 \mathrm{dex} / r_{\mathrm{e}}$. This value is slightly higher than the one derived for the galaxies without evidence of this inner drop in the abundances, which is $\alpha_{\mathrm{O} / \mathrm{H}}=-0.06 \mathrm{dex} / r_{\mathrm{e}}$ and $\sigma=0.05 \mathrm{dex} / r_{\mathrm{e}}$. A KS test suggested that the two distributions are different $(P$ value of $3 \%$ ), consistent with a radial movement of the gas as the cause of this feature.

Finally, $37 \%$ of the galaxies displaying this inner abundance drop neither showed evidence of a star-forming ring nor of a bar. This suggests that another mechanism related to radial movements is responsible for causing this feature.

To shed some further light on this question, we investigated the presence of this feature depending on the integrated stellar mass of the galaxies. We conclude that the galaxies displaying the strongest oxygen abundance inner drop are the most massive ones (Fig. 9), suggesting that stellar mass plays a key role in shaping the inner abundance profiles.

In summary, this is the first study that analysed the oxygen abundance distribution for a sample of face-on spiral galaxies spaxel by spaxel. Our final sample of 122 galaxies provided more than 185000 oxygen abundance values ( $~ 8200$ of them beyond two disc effective radii) to carry out the analysis. The results were compared to those obtained following the classical procedure of detecting $\mathrm{H}$ II regions, leading to equivalent results that point to the same conclusions: (i) the existence of a common abundance gradient, independent of other properties of galaxies, in particular the presence of bars, which seems not to have the flattening effect predicted by numerical simulations; (ii) the existence of a flattening of the abundance gradient in the outer regions of discs, which seems to be a common property of disc galaxies; and (iii) the existence of a drop of the abundance in the inner regions of disc galaxies, only visible in the most massive ones and most probably associated to radial movements of the gas (sometimes a bar or a circumnuclear star-forming ring). These results support the scenario in which disc galaxies present an overall inside-out growth. However, clear deviations were shown with respect to this simple scenario that affect the abundance profiles in both the innermost and outermost regions of galaxies.

The agreement between the two methods arises because the spaxel size of the CALIFA datacubes is of the order of the size of a typical H II region. However, this is not expected for IFS data with better spatial resolution and a smaller spaxel size, where different areas of the H II regions can be resolved and even abundance gradients can be found. Different procedures that can accomodate data that are capable of resolving $\mathrm{H}$ II regions may be needed.

Acknowledgements. This study makes use of the data provided by the Calar Alto Legacy Integral Field Area (CALIFA) survey (http://califa.caha. es/) based on observations collected at the Centro Astronómico Hispano Alemán (CAHA) at Calar Alto, operated jointly by the Max-Planck-Institut für Astronomie and the Instituto de Astrofísica de Andalucía (CSIC). CALIFA is the first legacy survey being performed at Calar Alto. The CALIFA collaboration would like to thank the IAA-CSIC and MPIA-MPG as major partners of the observatory, and CAHA itself, for the unique access to telescope time and support in manpower and infrastructures. The CALIFA collaboration also thanks the CAHA staff for the dedication to this project. We would like to thank the anonymous referee for comments that helped to improve the presentation of our results. We acknowledge financial support from the Spanish Ministerio de Economía y Competitividad (MINECO) via grant AYA2012-31935, and from the "Junta de Andalucía" local government through the FQM-108 project. We also acknowledge support to the ConaCyt funding program 180125. Y.A. acknowledges finantial support from the Ramón y Cajal programme (RyC-2011-09461). Y.A. and A.I.D. acknowledge support from the project AYA2013-47742-C4-3-P from the Spanish MINECO, as well as the "Study of Emission-Line Galaxies with Integral-Field Spectroscopy" (SELGIFS) programme, funded by the EU (FP7PEOPLE-2013-IRSES-612701). Support for L.G. is provided by the Ministry of Economy, Development, and Tourism's Millennium Science Initiative through grant IC120009, awarded to The Millennium Institute of Astrophysics, MAS. LG acknowledges support by CONICYT through FONDECYT grant 3140566. R.M.G.D. acknowledges support from the Spanish grant AYA2014-57490-P, and from the "Junta de Andalucía" P12-FQM2828 project. RAM thanks the Spanish program of International Campus of Excellence Moncloa (CEI). IM and A.d.O. acknowledge support from the Spanish MINECO grant AYA2013-42227P. JMA acknowledges support from the European Research Council Starting Grant (SEDmorph, P.I. V. Wild). Support for MM has been provided by DGICYT grant AYA2013-47742-C4-4-P. PSB acknowledges support from the Ramón y Cajal programme, grant ATA2010-21322-C03-02 from the Spanish MINECO. CJW acknowledges support through the Marie Curie Career Grant Integration 303912. This research makes use of python (http://www.python.org), of Matplotlib (Hunter 2007), a suite of open-source python modules that provides a framework for creating scientific plots, and Astropy, a community-developed core Python package for Astronomy (Astropy Collaboration et al. 2013).

\section{References}

Abazajian, K. N., Adelman-McCarthy, J. K., Agüeros, M. A., et al. 2009, ApJS, 182,543

Aguerri, J. A. L., Méndez-Abreu, J., \& Corsini, E. M. 2009, A\&A, 495, 491

Aller, L. H., ed. 1984, Physics of thermal gaseous nebulae, Astrophys. Space Sc.

Lib. (Dordrecht, Holland: D. Reidel Publishing Company), 112

Alloin, D., Collin-Souffrin, S., Joly, M., \& Vigroux, L. 1979, A\&A, 78, 200

Ascasibar, Y., Gavilán, M., Pinto, N., et al. 2015, MNRAS, 448, 2126

Astropy Collaboration, Robitaille, T. P., Tollerud, E. J., et al. 2013, A\&A, 558, A33

Athanassoula, E. 1992, MNRAS, 259, 345

Bacon, R., Copin, Y., Monnet, G., et al. 2001, MNRAS, 326, 23

Baldwin, J. A., Phillips, M. M., \& Terlevich, R. 1981, PASP, 93, 5

Balser, D. S., Rood, R. T., Bania, T. M., \& Anderson, L. D. 2011, ApJ, 738, 27

Barden, M., Rix, H.-W., Somerville, R. S., et al. 2005, ApJ, 635, 959

Barrera-Ballesteros, J. K., Sánchez, S. F., García-Lorenzo, B., et al. 2015, A\&A, 579, A45

Belfiore, F., Maiolino, R., Bundy, K., et al. 2015, MNRAS, 449, 867

Belley, J., \& Roy, J.-R. 1992, ApJS, 78, 61

Belli, S., Jones, T., Ellis, R. S., \& Richard, J. 2013, ApJ, 772, 141

Bershady, M. A., Verheijen, M. A. W., Swaters, R. A., et al. 2010, ApJ, 716, 198

Bilitewski, T., \& Schönrich, R. 2012, MNRAS, 426, 2266

Bird, J. C., Kazantzidis, S., \& Weinberg, D. H. 2012, MNRAS, 420, 913

Boissier, S., \& Prantzos, N. 1999, MNRAS, 307, 857 
Boissier, S., \& Prantzos, N. 2000, MNRAS, 312, 398

Bovy, J., \& Rix, H.-W. 2013, ApJ, 779, 115

Bresolin, F., Ryan-Weber, E., Kennicutt, R. C., \& Goddard, Q. 2009, ApJ, 695, 580

Bresolin, F., Kennicutt, R. C., \& Ryan-Weber, E. 2012, ApJ, 750, 122

Bundy, K., Bershady, M. A., Law, D. R., et al. 2015, ApJ, 798, 7

Cappellari, M., \& Emsellem, E. 2004, PASP, 116, 138

Cappellari, M., Emsellem, E., Krajnović, D., et al. 2011, MNRAS, 413, 813

Cardelli, J. A., Clayton, G. C., \& Mathis, J. S. 1989, ApJ, 345, 245

Carraro, G., Geisler, D., Villanova, S., Frinchaboy, P. M., \& Majewski, S. R. 2007, A\&A, 476, 217

Cavichia, O., Mollá, M., Costa, R. D. D., \& Maciel, W. J. 2014, MNRAS, 437, 3688

Cheung, E., Conroy, C., Athanassoula, E., et al. 2015, ApJ, 807, 36

Chiappini, C., Matteucci, F., \& Romano, D. 2001, ApJ, 554, 1044

Cid Fernandes, R., Mateus, A., Sodré, L., Stasińska, G., \& Gomes, J. M. 2005 MNRAS, 358, 363

Cid Fernandes, R., Stasińska, G., Mateus, A., \& Vale Asari, N. 2011, MNRAS, 413, 1687

Cid Fernandes, R., Pérez, E., García Benito, R., et al. 2013, A\&A, 557, A86

Comte, G. 1975, A\&A, 39, 197

Croom, S. M., Lawrence, J. S., Bland-Hawthorn, J., et al. 2012, MNRAS, 421, 872

Dalcanton, J. J. 2007, ApJ, 658, 941

Daniel, K. J., \& Wyse, R. F. G. 2015, MNRAS, 447, 3576

Davé, R., Finlator, K., \& Oppenheimer, B. D. 2011, MNRAS, 416, 1354

Davé, R., Finlator, K., \& Oppenheimer, B. D. 2012, MNRAS, 421, 98

Davies, R. L., Kewley, L. J., Ho, I.-T., \& Dopita, M. A. 2014, MNRAS, 444, 3961

de Jong, R. S. 1996, A\&A, 313, 377

Debattista, V. P., Mayer, L., Carollo, C. M., et al. 2006, ApJ, 645, 209

Deharveng, L., Peña, M., Caplan, J., \& Costero, R. 2000, MNRAS, 311, 329

Denicoló, G., Terlevich, R., \& Terlevich, E. 2002, MNRAS, 330, 69

Diaz, A. I. 1989, in Evolutionary Phenomena in Galaxies, eds. J. E. Beckman \& B. E. J. Pagel (Cambridge University Press), 377

Dopita, M. A., \& Evans, I. N. 1986, ApJ, 307, 431

Dopita, M. A., Fischera, J., Sutherland, R. S., et al. 2006, ApJS, 167, 177

Dopita, M. A., Sutherland, R. S., Nicholls, D. C., Kewley, L. J., \& Vogt, F. P. A. 2013, ApJS, 208, 10 (D10)

Edmunds, M. G., \& Greenhow, R. M. 1995, MNRAS, 272, 241

Ellison, S. L., Patton, D. R., Simard, L., \& McConnachie, A. W. 2008, ApJ, 672, L107

Esteban, C., García-Rojas, J., Peimbert, M., et al. 2005, ApJ, 618, L95

Esteban, C., Carigi, L., Copetti, M. V. F., et al. 2013, MNRAS, 433, 382

Falcón-Barroso, J., Sánchez-Blázquez, P., Vazdekis, A., et al. 2011, A\&A, 532, A95

Ferguson, A. M. N., \& Clarke, C. J. 2001, MNRAS, 325, 781

Friedli, D. 1998, in Abundance Profiles: Diagnostic Tools for Galaxy History, eds. D. Friedli, M. Edmunds, C. Robert, \& L. Drissen, ASP Conf. Ser., 147, 287

Friedli, D., \& Benz, W. 1995, A\&A, 301, 649

Friedli, D., Benz, W., \& Kennicutt, R. 1994, ApJ, 430, L105

Fu, J., Hou, J. L., Yin, J., \& Chang, R. X. 2009, ApJ, 696, 668

Galbany, L., Stanishev, V., Mourão, A. M., et al. 2014, A\&A, 572, A38

García-Benito, R., Zibetti, S., Sánchez, S. F., et al. 2015, A\&A, 576, A135

Garnett, D. R. 2002, ApJ, 581, 1019

Garnett, D. R., Shields, G. A., Skillman, E. D., Sagan, S. P., \& Dufour, R. J. 1997, ApJ, 489, 63

Giovanelli, R., Haynes, M. P., Salzer, J. J., et al. 1994, AJ, 107, 2036

Goetz, M., \& Koeppen, J. 1992, A\&A, 262, 455

Gomes, J. M., Papaderos, P., Kehrig, C., et al. 2016, A\&A, in press, DOI: 10.1051/0004-6361/201525976

González Delgado, R. M., \& Pérez, E. 1997, ApJS, 108, 199

González Delgado, R. M., Cerviño, M., Martins, L. P., Leitherer, C., \& Hauschildt, P. H. 2005, MNRAS, 357, 945

González Delgado, R. M., Pérez, E., Cid Fernandes, R., et al. 2014, A\&A, 562, A47

González Delgado, R. M., García-Benito, R., Pérez, E., et al. 2015, A\&A, 581, A103

Hammer, F., Puech, M., Chemin, L., Flores, H., \& Lehnert, M. D. 2007, ApJ, 662,322

Hayden, M. R., Holtzman, J. A., Bovy, J., et al. 2014, AJ, 147, 116

Ho, L. C., Filippenko, A. V., \& Sargent, W. L. W. 1997, ApJ, 487, 579

Ho, I.-T., Kudritzki, R.-P., Kewley, L. J., et al. 2015, MNRAS, 448, 2030

Holmes, L., Spekkens, K., Sánchez, S. F., et al. 2015, MNRAS, 451, 4397

Hunter, J. D. 2007, Computing In Science \& Engineering, 9, 90

Huntley, J. M. 1978, ApJ, 225, L101

Husemann, B., Jahnke, K., Sánchez, S. F., et al. 2013, A\&A, 549, A87
Izotov, Y. I., Stasińska, G., Meynet, G., Guseva, N. G., \& Thuan, T. X. 2006, A\&A, 448, 955

Karachentsev, I. D., Karachentseva, V. E., Huchtmeier, W. K., \& Makarov, D. I. 2004, AJ, 127, 2031

Kauffmann, G., Heckman, T. M., Tremonti, C., et al. 2003, MNRAS, 346, 1055

Kehrig, C., Monreal-Ibero, A., Papaderos, P., et al. 2012, A\&A, 540, A11

Kelz, A., Verheijen, M. A. W., Roth, M. M., et al. 2006, PASP, 118, 129

Kennicutt, Jr., R. C., Keel, W. C., \& Blaha, C. A. 1989, AJ, 97, 1022

Kennicutt, Jr., R. C., Bresolin, F., \& Garnett, D. R. 2003, ApJ, 591, 801

Kewley, L. J., \& Dopita, M. A. 2002, ApJS, 142, 35

Kewley, L. J., Dopita, M. A., Sutherland, R. S., Heisler, C. A., \& Trevena, J. 2001, ApJ, 556, 121

Kobulnicky, H. A., \& Kewley, L. J. 2004, ApJ, 617, 240

Kobulnicky, H. A., \& Koo, D. C. 2000, ApJ, 545, 712

Koeppen, J. 1994, A\&A, 281, 26

Koleva, M., Prugniel, P., Bouchard, A., \& Wu, Y. 2009, A\&A, 501, 1269

Lacey, C. G., \& Fall, S. M. 1985, ApJ, 290, 154

Lara-López, M. A., Bongiovanni, A., Cepa, J., et al. 2010, A\&A, 519, A31

Lequeux, J., Peimbert, M., Rayo, J. F., Serrano, A., \& Torres-Peimbert, S. 1979, A\&A, 80, 155

Li, C., Wang, E., Lin, L., et al. 2015, ApJ, 804, 125

Lilliefors, H. W. 1967, J. Am. Statist. Assoc., 62, 399

Lopez, L. A., Krumholz, M. R., Bolatto, A. D., Prochaska, J. X., \& Ramirez-Ruiz, E. 2011, ApJ, 731, 91

López-Sánchez, Á. R. 2010, A\&A, 521, A63

López-Sánchez, Á. R., Dopita, M. A., Kewley, L. J., et al. 2012, MNRAS, 426, 2630

López-Sánchez, Á. R., Westmeier, T., Esteban, C., \& Koribalski, B. S. 2015, MNRAS, 450, 3381

MacArthur, L. A., Courteau, S., Bell, E., \& Holtzman, J. A. 2004, ApJS, 152, 175

Maiolino, R., Nagao, T., Grazian, A., et al. 2008, A\&A, 488, 463

Mannucci, F., Cresci, G., Maiolino, R., et al. 2009, MNRAS, 398, 1915

Mannucci, F., Cresci, G., Maiolino, R., Marconi, A., \& Gnerucci, A. 2010, MNRAS, 408, 2115

Marino, R. A., Gil de Paz, A., Castillo-Morales, A., et al. 2012, ApJ, 754, 61

Marino, R. A., Rosales-Ortega, F. F., Sánchez, S. F., et al. 2013, A\&A, 559, A114 (M13)

Marino, R. A., Gil de Paz, A., Sánchez, S. F., et al. 2016, A\&A, 585, A47

Marinova, I., \& Jogee, S. 2007, ApJ, 659, 1176

Mármol-Queraltó, E., Sánchez, S. F., Marino, R. A., et al. 2011, A\&A, 534, A8

Martin, P., \& Roy, J.-R. 1992, ApJ, 397, 463

Martin, P., \& Roy, J.-R. 1994, ApJ, 424, 599

Martin, P., \& Roy, J.-R. 1995, ApJ, 445, 161

Martín-Navarro, I., Vazdekis, A., La Barbera, F., et al. 2015, ApJ, 806, L31

Masters, K. L., Nichol, R. C., Hoyle, B., et al. 2011, MNRAS, 411, 2026

Matteucci, F., \& Francois, P. 1989, MNRAS, 239, 885

McGaugh, S. S. 1991, ApJ, 380, 140

Minchev, I., \& Famaey, B. 2010, ApJ, 722, 112

Minchev, I., Famaey, B., Combes, F., et al. 2011, A\&A, 527, A147

Minchev, I., Famaey, B., Quillen, A. C., et al. 2012, A\&A, 548, A126

Mollá, M., \& Díaz, A. I. 2005, MNRAS, 358, 521

Molla, M., Ferrini, F., \& Diaz, A. I. 1996, ApJ, 466, 668

Mollá, M., Ferrini, F., \& Díaz, A. I. 1997, ApJ, 475, 519

Mott, A., Spitoni, E., \& Matteucci, F. 2013, MNRAS, 435, 2918

Moustakas, J., Kennicutt, Jr., R. C., Tremonti, C. A., et al. 2010, ApJS, 190, 233

Muñoz-Mateos, J. C., Gil de Paz, A., Boissier, S., et al. 2007, ApJ, 658, 1006

Ocvirk, P., Pichon, C., Lançon, A., \& Thiébaut, E. 2006, MNRAS, 365, 46

Oey, M. S., \& Kennicutt, Jr., R. C. 1993, ApJ, 411, 137

Oey, M. S., Parker, J. S., Mikles, V. J., \& Zhang, X. 2003, AJ, 126, 2317

Oppenheimer, B. D., \& Davé, R. 2008, MNRAS, 387, 577

Oppenheimer, B. D., Davé, R., Kereš, D., et al. 2010, MNRAS, 406, 2325

Osterbrock, D. E. 1989, Astrophysics of gaseous nebulae and active galactic nuclei (Mille Valley, CA: University Science Books)

Pagel, B. E. J., Edmunds, M. G., Blackwell, D. E., Chun, M. S., \& Smith, G. 1979, MNRAS, 189, 95

Pagel, B. E. J., Simonson, E. A., Terlevich, R. J., \& Edmunds, M. G. 1992, MNRAS, 255, 325

Papaderos, P., Gomes, J. M., Vílchez, J. M., et al. 2013, A\&A, 555, L1

Patterson, M. T., Walterbos, R. A. M., Kennicutt, R. C., Chiappini, C., \& Thilker, D. A. 2012, MNRAS, 422, 401

Pedicelli, S., Bono, G., Lemasle, B., et al. 2009, A\&A, 504, 81

Peimbert, M. 1979, in The Large-Scale Characteristics of the Galaxy, ed. W. B. Burton, IAU Symp., 84, 307

Peimbert, M., \& Costero, R. 1969, Boletin de los Observatorios Tonantzintla y Tacubaya, 5, 3

Pérez, E., Cid Fernandes, R., González Delgado, R. M., et al. 2013, ApJ, 764, L1 
Pérez-Montero, E. 2014, MNRAS, 441, 2663

Pérez-Montero, E., \& Díaz, A. I. 2005, MNRAS, 361, 1063

Pettini, M., \& Pagel, B. E. J. 2004, MNRAS, 348, L59 (PP04)

Pilkington, K., Few, C. G., Gibson, B. K., et al. 2012, A\&A, 540, A56

Pilyugin, L. S. 2000, A\&A, 362, 325

Pilyugin, L. S., \& Thuan, T. X. 2005, ApJ, 631, 231

Pilyugin, L. S., Ferrini, F., \& Shkvarun, R. V. 2003, A\&A, 401, 557

Pilyugin, L. S., Vílchez, J. M., \& Contini, T. 2004, A\&A, 425, 849

Pilyugin, L. S., Vílchez, J. M., \& Thuan, T. X. 2010, ApJ, 720,1738 (P10)

Portinari, L., \& Chiosi, C. 2000, A\&A, 355, 929

Prantzos, N., \& Boissier, S. 2000, MNRAS, 313, 338

Qu, Y., Di Matteo, P., Lehnert, M. D., van Driel, W., \& Jog, C. J. 2011, A\&A, 535, A5

Quillen, A. C., Minchev, I., Bland-Hawthorn, J., \& Haywood, M. 2009, MNRAS, 397, 1599

Quireza, C., Rood, R. T., Bania, T. M., Balser, D. S., \& Maciel, W. J. 2006, ApJ, 653,1226

Rich, J. A., Torrey, P., Kewley, L. J., Dopita, M. A., \& Rupke, D. S. N. 2012, ApJ, 753, 5

Roberts, M. S., \& Haynes, M. P. 1994, ARA\&A, 32, 115

Rosales-Ortega, F. F., Kennicutt, R. C., Sánchez, S. F., et al. 2010, MNRAS, 405, 735

Rosales-Ortega, F. F., Díaz, A. I., Kennicutt, R. C., \& Sánchez, S. F. 2011, MNRAS, 415, 2439

Rosales-Ortega, F. F., Sánchez, S. F., Iglesias-Páramo, J., et al. 2012, ApJ, 756, L31

Roth, M. M., Kelz, A., Fechner, T., et al. 2005, PASP, 117, 620

Roškar, R., Debattista, V. P., Quinn, T. R., \& Wadsley, J. 2012, MNRAS, 426, 2089

Roy, J.-R., \& Walsh, J. R. 1997, MNRAS, 288, 715

Rudolph, A. L., Fich, M., Bell, G. R., et al. 2006, ApJS, 162, 346

Ruiz-Lara, T., Pérez, I., Florido, E., et al. 2016, MNRAS, 456, L35

Sackett, P. D. 1997, ApJ, 483, 103

Sánchez, S. F., Rosales-Ortega, F. F., Kennicutt, R. C., et al. 2011, MNRAS, 410, 313

Sánchez, S. F., Kennicutt, R. C., Gil de Paz, A., et al. 2012a, A\&A, 538, A8

Sánchez, S. F., Rosales-Ortega, F. F., Marino, R. A., et al. 2012b, A\&A, 546, A2

Sánchez, S. F., Rosales-Ortega, F. F., Jungwiert, B., et al. 2013, A\&A, 554, A58

Sarzi, M., Falcón-Barroso, J., Davies, R. L., et al. 2006, MNRAS, 366, 1151

Sánchez, S. F., Rosales-Ortega, F. F., Iglesias-Páramo, J., et al. 2014, A\&A, 563, A49 (S14)

Scannapieco, C., Tissera, P. B., White, S. D. M., \& Springel, V. 2008, MNRAS, 389,1137

Sánchez, S. F., Pérez, E., Rosales-Ortega, F. F., et al. 2015a, A\&A, 574, A47

Sánchez, S. F., Pérez, E., Sánchez-Blázquez, P., et al. 2015b, Rev. Mex. Astron. Astrofis., submitted [arXiv: 1509.08552]
Sánchez-Blázquez, P., Courty, S., Gibson, B. K., \& Brook, C. B. 2009, MNRAS, 398, 591

Sánchez-Blázquez, P., Rosales-Ortega, F. F., Méndez-Abreu, J., et al. 2014, A\&A, 570, A6

Scannapieco, C., White, S. D. M., Springel, V., \& Tissera, P. B. 2009, MNRAS, 396, 696

Schönrich, R., \& Binney, J. 2009, MNRAS, 396, 203

Searle, L. 1971, ApJ, 168, 327

Sellwood, J. A., \& Binney, J. J. 2002, MNRAS, 336, 785

Sellwood, J. A., \& Wilkinson, A. 1993, Rep. Progr. Phys., 56, 173

Shaver, P. A., McGee, R. X., Newton, L. M., Danks, A. C., \& Pottasch, S. R. 1983, MNRAS, 204, 53

Sheth, K., Elmegreen, D. M., Elmegreen, B. G., et al. 2008, ApJ, 675, 1141

Singh, R., van de Ven, G., Jahnke, K., et al. 2013, A\&A, 558, A43

Skillman, E. D., Kennicutt, R. C., \& Hodge, P. W. 1989, ApJ, 347, 875

Smith, H. E. 1975, ApJ, 199, 591

Spitoni, E., Matteucci, F., \& Marcon-Uchida, M. M. 2013, A\&A, 551, A123

Stasińska, G. 1978, A\&A, 66, 257

Stasińska, G., Vale Asari, N., Cid Fernandes, R., et al. 2008, MNRAS, 391 , L29

Taylor, V. A., Jansen, R. A., Windhorst, R. A., Odewahn, S. C., \& Hibbard, J. E. 2005, ApJ, 630, 784

Tremonti, C. A., Heckman, T. M., Kauffmann, G., et al. 2004, ApJ, 613, 898

Trujillo, I., Rudnick, G., Rix, H.-W., et al. 2004, ApJ, 604, 521

Trujillo, I., Förster Schreiber, N. M., Rudnick, G., et al. 2006, ApJ, 650, 18

Tsujimoto, T., Yoshii, Y., Nomoto, K., \& Shigeyama, T. 1995, A\&A, 302, 704

van der Kruit, P. C., \& Freeman, K. C. 2011, ARA\&A, 49, 301

van Zee, L., Salzer, J. J., Haynes, M. P., O’Donoghue, A. A., \& Balonek, T. J. 1998, AJ, 116, 2805

Vazdekis, A., Sánchez-Blázquez, P., Falcón-Barroso, J., et al. 2010, MNRAS, 404, 1639

Veilleux, S., \& Osterbrock, D. E. 1987, ApJS, 63, 295

Veilleux, S., Cecil, G., \& Bland-Hawthorn, J. 2005, ARA\&A, 43, 769

Vila-Costas, M. B., \& Edmunds, M. G. 1992, MNRAS, 259, 121

Vilchez, J. M., \& Esteban, C. 1996, MNRAS, 280, 720

Vlajić, M., Bland-Hawthorn, J., \& Freeman, K. C. 2009, ApJ, 697, 361

Vlajić, M., Bland-Hawthorn, J., \& Freeman, K. C. 2011, ApJ, 732, 7

Walcher, C. J., Wisotzki, L., Bekeraité, S., et al. 2014, A\&A, 569, A1

Werk, J. K., Putman, M. E., Meurer, G. R., et al. 2010, ApJ, 715, 656

Werk, J. K., Putman, M. E., Meurer, G. R., \& Santiago-Figueroa, N. 2011, ApJ, 735,71

Wilkinson, D. M., Maraston, C., Thomas, D., et al. 2015, MNRAS, 449, 328

Yong, D., Carney, B. W., Teixera de Almeida, M. L., \& Pohl, B. L. 2006, AJ, 131,2256

Zahid, H. J., Dima, G. I., Kudritzki, R.-P., et al. 2014, ApJ, 791, 130

Zaritsky, D., Kennicutt, Jr., R. C., \& Huchra, J. P. 1994, ApJ, 420, 87

Zurita, A., Relaño, M., Beckman, J. E., \& Knapen, J. H. 2004, A\&A, 413, 73 


\section{Appendix A: Fundamental parameters and oxygen abundance information}

In this section we present a table with general information and derived oxygen abundance information for all the galaxies in the sample. The meaning of each column is indicated in the notes below.

Table A.1. Fundamental properties and oxygen abundance information.

\begin{tabular}{|c|c|c|c|c|c|c|c|}
\hline Name & $\begin{array}{l}\text { Morph } \\
\text { type } \\
\text { (b) }\end{array}$ & $\begin{array}{c}M_{g} \\
{[\mathrm{mag}]} \\
\text { (c) }\end{array}$ & $\begin{array}{c}\log _{10} \text { Mass } \\
{\left[M_{\odot}\right]} \\
\text { (d) }\end{array}$ & $\begin{array}{c}r_{\mathrm{e}} \\
{[\mathrm{kpc}]} \\
\text { (e) }\end{array}$ & $\begin{array}{c}{[\mathrm{O} / \mathrm{H}]_{r_{\mathrm{e}}}} \\
{[\mathrm{dex}]} \\
\text { (f) }\end{array}$ & $\begin{array}{c}\alpha_{\mathrm{O} / \mathrm{H}} \\
{\left[\mathrm{dex} / r_{\mathrm{e}}\right]} \\
(\mathrm{g})\end{array}$ & c.c. \\
\hline IC 0159 & SBdm & -19.99 & 9.82 & 5.42 & $8.40 \pm 0.06$ & $-0.03 \pm 0.03$ & 0.664 \\
\hline IC 0674 & SBab & -21.43 & 10.91 & 11.55 & $8.44 \pm 0.07$ & $-0.100 \pm 0.015$ & 0.871 \\
\hline IC 0776 & $\mathrm{Sdm}$ & -19.11 & 9.27 & 8.38 & $8.27 \pm 0.05$ & $-0.08 \pm 0.04$ & 0.855 \\
\hline IC 1256 & $\mathrm{SABb}$ & -20.73 & 10.37 & 6.82 & $8.58 \pm 0.06$ & $-0.146 \pm 0.015$ & 0.983 \\
\hline IC 1683 & $\mathrm{SABb}$ & -20.45 & 10.51 & 4.78 & $8.57 \pm 0.05$ & $-0.08 \pm 0.05$ & 0.972 \\
\hline IC 4566 & $\mathrm{SBb}$ & -21.27 & 10.94 & 10.30 & $8.54 \pm 0.04$ & $0.00 \pm 0.04$ & 0.007 \\
\hline MCG-01-10-019 & $\mathrm{SABbc}$ & -20.62 & 10.21 & 12.04 & $8.40 \pm 0.06$ & $-0.12 \pm 0.04$ & 0.925 \\
\hline NGC 0001 & $\mathrm{Sbc}$ & -21.07 & 10.80 & 5.99 & $8.56 \pm 0.06$ & $-0.020 \pm 0.014$ & 0.470 \\
\hline NGC 0036 & $\mathrm{SBb}$ & -21.77 & 10.90 & 14.57 & $8.53 \pm 0.07$ & $-0.04 \pm 0.03$ & 0.890 \\
\hline NGC 0160 & $\mathrm{Sa}$ & -21.55 & 11.06 & 11.44 & $8.53 \pm 0.08$ & $0.05 \pm 0.07$ & 0.851 \\
\hline NGC 0165 & $\mathrm{SBb}$ & -21.09 & 10.59 & 13.04 & $8.51 \pm 0.04$ & $-0.07 \pm 0.04$ & 0.966 \\
\hline NGC 0171 & $\mathrm{SBb}$ & -21.26 & 10.72 & 6.98 & $8.58 \pm 0.06$ & $-0.072 \pm 0.016$ & 0.894 \\
\hline NGC 0214 & SABbc & -21.60 & 10.85 & 6.66 & $8.58 \pm 0.05$ & $-0.059 \pm 0.012$ & 0.991 \\
\hline NGC 0234 & $\mathrm{SABc}$ & -21.40 & 10.65 & 6.36 & $8.58 \pm 0.05$ & $-0.07 \pm 0.02$ & 0.982 \\
\hline NGC 0237 & $\mathrm{SBc}$ & -20.72 & 10.28 & 4.27 & $8.56 \pm 0.05$ & $-0.066 \pm 0.011$ & 0.977 \\
\hline NGC 0257 & $\mathrm{Sc}$ & -21.60 & 10.80 & 8.67 & $8.57 \pm 0.05$ & $-0.074 \pm 0.014$ & 0.913 \\
\hline NGC 0309 & SBcd & -22.38 & 10.83 & 13.37 & $8.50 \pm 0.05$ & $-0.15 \pm 0.03$ & 0.986 \\
\hline NGC 0477 & SABbc & -21.24 & 10.50 & 14.26 & $8.43 \pm 0.08$ & $-0.19 \pm 0.06$ & 0.990 \\
\hline NGC 0496 & Scd & -20.93 & 10.35 & 10.86 & $8.46 \pm 0.06$ & $-0.14 \pm 0.03$ & 0.970 \\
\hline NGC 0570 & $\mathrm{SBb}$ & -21.24 & 10.96 & 8.61 & $8.47 \pm 0.03$ & $-0.10 \pm 0.03$ & 0.965 \\
\hline NGC 0716 & $\mathrm{SABb}$ & -20.72 & 10.58 & 5.54 & $8.50 \pm 0.06$ & $-0.09 \pm 0.02$ & 0.971 \\
\hline NGC 0768 & $\mathrm{SBc}$ & -21.33 & 10.55 & 10.40 & $8.41 \pm 0.08$ & $-0.013 \pm 0.014$ & 0.469 \\
\hline NGC 0776 & $\mathrm{SBb}$ & -21.26 & 10.70 & 6.88 & $8.56 \pm 0.06$ & $-0.030 \pm 0.013$ & 0.822 \\
\hline NGC 0787 & $\mathrm{Sa}$ & -21.30 & 10.96 & 6.68 & $8.50 \pm 0.06$ & $-0.04 \pm 0.02$ & 0.965 \\
\hline NGC 0873 & Scd & -21.17 & 10.41 & 4.32 & $8.53 \pm 0.03$ & $-0.061 \pm 0.011$ & 0.995 \\
\hline NGC 0941 & Scd & -19.13 & 9.35 & 2.87 & $8.40 \pm 0.06$ & $-0.034 \pm 0.019$ & 0.794 \\
\hline NGC 0976 & $\mathrm{Sbc}$ & -21.50 & 10.80 & 5.72 & $8.48 \pm 0.00$ & $-0.068 \pm 0.018$ & 0.753 \\
\hline NGC 0991 & SABcd & -19.38 & 9.61 & 3.65 & $8.41 \pm 0.06$ & $-0.09 \pm 0.02$ & 0.851 \\
\hline NGC 1070 & $\mathrm{Sb}$ & -21.73 & 11.00 & 7.08 & $8.53 \pm 0.05$ & $-0.006 \pm 0.014$ & 0.470 \\
\hline NGC 1093 & $\mathrm{SBbc}$ & -20.82 & 10.75 & 7.27 & $8.52 \pm 0.06$ & $-0.090 \pm 0.015$ & 0.979 \\
\hline NGC 1094 & $\mathrm{SABb}$ & -21.55 & 10.72 & 7.45 & $8.50 \pm 0.05$ & $-0.084 \pm 0.012$ & 0.950 \\
\hline NGC 1659 & SABbc & -21.15 & 10.59 & 6.35 & $8.50 \pm 0.06$ & $-0.094 \pm 0.012$ & 0.956 \\
\hline NGC 1667 & $\mathrm{SBbc}$ & -22.02 & 10.80 & 4.91 & $8.56 \pm 0.04$ & $-0.033 \pm 0.011$ & 0.936 \\
\hline NGC 2253 & $\mathrm{SBbc}$ & -21.06 & 10.47 & 2.53 & $8.57 \pm 0.06$ & $-0.011 \pm 0.012$ & 0.558 \\
\hline NGC 2347 & SABbc & -21.66 & 10.72 & 6.31 & $8.53 \pm 0.06$ & $-0.108 \pm 0.012$ & 0.998 \\
\hline NGC 2449 & SABab & -20.98 & 10.83 & 5.85 & $8.56 \pm 0.05$ & $-0.002 \pm 0.011$ & 0.252 \\
\hline NGC 2486 & SBab & -20.64 & 10.58 & 9.55 & $8.49 \pm 0.05$ & $-0.07 \pm 0.03$ & 0.989 \\
\hline NGC 2487 & $\mathrm{SBb}$ & -21.68 & 10.77 & 12.04 & $8.54 \pm 0.05$ & $-0.007 \pm 0.015$ & 0.078 \\
\hline NGC 2530 & SABd & -20.96 & 10.19 & 9.78 & $8.40 \pm 0.06$ & $-0.09 \pm 0.03$ & 0.994 \\
\hline NGC 2540 & SBbc & -21.15 & 10.50 & 8.28 & $8.49 \pm 0.06$ & $-0.063 \pm 0.013$ & 0.753 \\
\hline NGC 2604 & SBd & -19.97 & 9.64 & 4.09 & $8.35 \pm 0.06$ & $-0.037 \pm 0.013$ & 0.216 \\
\hline NGC 2639 & $\mathrm{Sa}$ & -21.59 & 11.18 & 4.88 & $8.57 \pm 0.06$ & $-0.037 \pm 0.019$ & 0.723 \\
\hline NGC 2730 & SBcd & -20.56 & 10.11 & 7.83 & $8.46 \pm 0.05$ & $-0.049 \pm 0.014$ & 0.945 \\
\hline NGC 2805 & $\mathrm{Sc}$ & -20.30 & 10.03 & 7.86 & $8.44 \pm 0.04$ & $-0.11 \pm 0.03$ & 0.991 \\
\hline NGC 2906 & $\mathrm{Sbc}$ & -20.14 & 10.39 & 3.41 & $8.60 \pm 0.04$ & $-0.034 \pm 0.011$ & 0.929 \\
\hline NGC 2916 & $\mathrm{Sbc}$ & -21.57 & 10.75 & 8.08 & $8.56 \pm 0.04$ & $-0.100 \pm 0.013$ & 0.986 \\
\hline NGC 3057 & SBdm & -19.01 & 9.18 & 6.02 & $8.27 \pm 0.08$ & $-0.12 \pm 0.07$ & 0.997 \\
\hline NGC 3106 & $\mathrm{Sab}$ & -22.16 & 11.31 & 13.31 & $8.50 \pm 0.05$ & $-0.06 \pm 0.05$ & 0.922 \\
\hline NGC 3381 & SBd & -19.74 & 9.68 & 3.07 & $8.50 \pm 0.03$ & $-0.057 \pm 0.012$ & 0.972 \\
\hline NGC 3614 & $\mathrm{SABbc}$ & -20.72 & 10.21 & 9.56 & $8.42 \pm 0.06$ & $-0.22 \pm 0.05$ & 0.999 \\
\hline NGC 3687 & $\mathrm{SBb}$ & -20.47 & 10.28 & 4.72 & $8.50 \pm 0.06$ & $-0.156 \pm 0.010$ & 0.989 \\
\hline NGC 3811 & SBbc & -20.92 & 10.45 & 5.39 & $8.57 \pm 0.04$ & $-0.076 \pm 0.010$ & 0.923 \\
\hline NGC 4047 & $\mathrm{Sbc}$ & -21.41 & 10.68 & 5.05 & $8.57 \pm 0.05$ & $-0.104 \pm 0.012$ & 0.972 \\
\hline NGC 4185 & SABbc & -21.35 & 10.72 & 10.31 & $8.55 \pm 0.05$ & $-0.082 \pm 0.012$ & 0.898 \\
\hline
\end{tabular}

Notes. (a) The galaxy name; (b) the morphological type; (c) $g$-band absolute magnitude; (d) the logarithm of the integrated stellar mass in units of solar masses; (e) the disc effective radius $\left(r_{\mathrm{e}}\right.$, in kpc); (f) the oxygen abundance value at one effective radius; $(\mathrm{g})$ the slope of the oxygen abundance gradient measured between 0.5 and $2.0 r_{\mathrm{e}}$; and (h) the correlation coefficient of the linear fit (c.c.). 
Table A.1. continued.

\begin{tabular}{llcccccc}
\hline \hline Name & Morph & $M_{g}$ & $\log _{10}$ Mass & $r_{\mathrm{e}}$ & {$[\mathrm{O} / \mathrm{H}]_{r_{\mathrm{e}}}$} & $\alpha_{\mathrm{O} / \mathrm{H}}$ & c.c. \\
& type & {$[\mathrm{mag}]$} & {$\left[M_{\odot}\right]$} & {$[\mathrm{kpc}]$} & {$[\mathrm{dex}]$} & {$\left[\mathrm{dex} / r_{\mathrm{e}}\right]$} &
\end{tabular}

(a)

(b)

(c)

(d)

(e) (f)

(g)

(h)

\begin{tabular}{|c|c|c|c|c|c|c|c|}
\hline NGC 4210 & $\mathrm{SBb}$ & -20.46 & 10.28 & 4.42 & $8.58 \pm 0.04$ & $-0.085 \pm 0.011$ & 0.935 \\
\hline NGC 4470 & $\mathrm{Sc}$ & -20.37 & 9.98 & 3.46 & $8.42 \pm 0.02$ & $-0.017 \pm 0.008$ & 0.037 \\
\hline NGC 4961 & SBcd & -19.97 & 9.68 & 3.87 & $8.42 \pm 0.05$ & $-0.095 \pm 0.012$ & 0.973 \\
\hline NGC 5000 & $\mathrm{SBbc}$ & -21.32 & 10.66 & 8.16 & $8.55 \pm 0.06$ & $-0.065 \pm 0.012$ & 0.855 \\
\hline NGC 5016 & $\mathrm{Sbc}$ & -20.61 & 10.23 & 4.64 & $8.54 \pm 0.05$ & $-0.112 \pm 0.013$ & 0.974 \\
\hline NGC 5056 & $\mathrm{SABc}$ & -21.43 & 10.45 & 8.65 & $8.44 \pm 0.05$ & $-0.125 \pm 0.010$ & 0.981 \\
\hline NGC 5157 & SBab & -21.81 & 11.27 & 10.06 & $8.55 \pm 0.07$ & $-0.01 \pm 0.02$ & 0.442 \\
\hline NGC 5205 & SBbc & -19.66 & 9.88 & 3.79 & $8.52 \pm 0.06$ & $-0.04 \pm 0.02$ & 0.690 \\
\hline NGC 5320 & SABbc & -20.72 & 10.29 & 8.17 & $8.50 \pm 0.07$ & $-0.028 \pm 0.018$ & 0.985 \\
\hline NGC 5376 & $\mathrm{SABb}$ & -20.31 & 10.47 & 3.76 & $8.60 \pm 0.05$ & $-0.037 \pm 0.013$ & 0.685 \\
\hline NGC 5378 & $\mathrm{SBb}$ & -20.64 & 10.62 & 7.36 & $8.53 \pm 0.04$ & $-0.01 \pm 0.03$ & 0.314 \\
\hline NGC 5406 & $\mathrm{SBb}$ & -21.94 & 11.19 & 9.72 & $8.53 \pm 0.06$ & $-0.03 \pm 0.02$ & 0.863 \\
\hline NGC 5480 & Scd & -20.30 & 10.14 & 3.13 & $8.57 \pm 0.04$ & $-0.046 \pm 0.012$ & 0.989 \\
\hline NGC 5520 & Sbc & -19.77 & 9.85 & 3.39 & $8.49 \pm 0.04$ & $-0.089 \pm 0.009$ & 0.982 \\
\hline NGC 5533 & $\mathrm{Sab}$ & -21.91 & 11.23 & 14.16 & $8.49 \pm 0.05$ & $-0.06 \pm 0.03$ & 0.811 \\
\hline NGC 5622 & $\mathrm{Sbc}$ & -20.41 & 10.22 & 6.54 & $8.53 \pm 0.08$ & $-0.039 \pm 0.014$ & 0.813 \\
\hline NGC 5633 & Sbc & -20.44 & 10.27 & 2.50 & $8.60 \pm 0.03$ & $-0.064 \pm 0.007$ & 0.917 \\
\hline NGC 5656 & $\mathrm{Sb}$ & -21.11 & 10.62 & 4.28 & $8.55 \pm 0.05$ & $-0.097 \pm 0.011$ & 0.984 \\
\hline NGC 5657 & $\mathrm{SBbc}$ & -20.48 & 10.28 & 6.95 & $8.47 \pm 0.07$ & $-0.038 \pm 0.012$ & 0.757 \\
\hline NGC 5665 & $\mathrm{SABc}$ & -20.66 & 10.22 & 4.72 & $8.50 \pm 0.05$ & $-0.04 \pm 0.03$ & 0.904 \\
\hline NGC 5720 & SBbc & -21.79 & 10.85 & 12.32 & $8.53 \pm 0.05$ & $-0.130 \pm 0.017$ & 0.920 \\
\hline NGC 5732 & Sbc & -20.06 & 9.93 & 6.52 & $8.47 \pm 0.06$ & $-0.18 \pm 0.03$ & 0.999 \\
\hline NGC 5735 & SBbc & -21.02 & 10.38 & 8.05 & $8.49 \pm 0.06$ & $-0.16 \pm 0.02$ & 0.997 \\
\hline NGC 5772 & Sab & -21.56 & 10.94 & 9.39 & $8.52 \pm 0.06$ & $-0.06 \pm 0.02$ & 0.995 \\
\hline NGC 5829 & $\mathrm{Sc}$ & -21.36 & 10.53 & 10.77 & $8.43 \pm 0.05$ & $-0.074 \pm$ & 0.927 \\
\hline NGC 5888 & $\mathrm{SBb}$ & -22.06 & 11.29 & 11.45 & $8.54 \pm 0.06$ & $-0.02 \pm 0.02$ & 0.690 \\
\hline NGC 5947 & SBbc & -21.02 & 10.37 & 7.70 & $8.49 \pm 0.05$ & $-0.100 \pm 0.013$ & 0.983 \\
\hline NGC 5957 & $\mathrm{SBb}$ & -20.34 & 10.28 & 4.69 & $8.56 \pm 0.05$ & $-0.06 \pm 0.02$ & 0.958 \\
\hline NGC 6004 & SBbc & -21.30 & 10.71 & 7.96 & $8.53 \pm 0.06$ & $-0.033 \pm 0.020$ & 0.784 \\
\hline NGC 6063 & $\mathrm{Sbc}$ & -20.05 & 10.06 & 6.34 & $8.48 \pm 0.05$ & $-0.17 \pm 0.02$ & 0.986 \\
\hline NGC 6154 & SBab & -21.63 & 10.95 & 9.42 & $8.52 \pm 0.07$ & $-0.003 \pm 0.019$ & 0.028 \\
\hline NGC 6155 & $\mathrm{Sc}$ & -20.28 & 10.12 & 3.47 & $8.55 \pm 0.03$ & $-0.068 \pm 0.009$ & 0.973 \\
\hline NGC 6301 & $\mathrm{Sbc}$ & -22.23 & 11.02 & 15.71 & $8.51 \pm 0.04$ & $-0.063 \pm 0.014$ & 0.879 \\
\hline NGC 6941 & $\mathrm{SBb}$ & -21.85 & 10.93 & 12.10 & $8.54 \pm 0.06$ & $-0.05 \pm 0.03$ & 0.719 \\
\hline NGC 7321 & SBbc & -22.06 & 10.81 & 9.58 & $8.52 \pm 0.05$ & $-0.082 \pm 0.011$ & 0.964 \\
\hline NGC 7364 & Sab & -21.41 & 10.89 & 5.92 & $8.57 \pm 0.07$ & $-0.028 \pm 0.013$ & 0.777 \\
\hline NGC 7466 & Sbc & -21.29 & 10.83 & 12.32 & $8.47 \pm 0.05$ & $-0.085 \pm 0.018$ & 0.933 \\
\hline NGC 7489 & Sbc & -21.79 & 10.40 & 10.59 & $8.41 \pm 0.06$ & $-0.150 \pm 0.013$ & 0.970 \\
\hline NGC 7591 & SBbc & -21.35 & 10.75 & 8.43 & $8.52 \pm 0.07$ & $-0.084 \pm 0.020$ & 0.960 \\
\hline NGC 7625 & $\mathrm{Sa}$ & -19.71 & 10.07 & 2.00 & $8.54 \pm 0.04$ & $-0.033 \pm 0.011$ & 0.889 \\
\hline NGC 7653 & $\mathrm{Sb}$ & -21.11 & 10.50 & 6.17 & $8.52 \pm 0.05$ & $-0.090 \pm 0.015$ & 0.995 \\
\hline NGC 7691 & $\mathrm{SBbc}$ & -20.97 & 10.21 & 8.94 & $8.45 \pm 0.07$ & $-0.10 \pm 0.04$ & 0.967 \\
\hline NGC 7716 & $\mathrm{Sb}$ & -20.56 & 10.32 & 4.46 & $8.49 \pm 0.04$ & $-0.025 \pm 0.010$ & 0.850 \\
\hline NGC 7782 & $\mathrm{Sb}$ & -21.94 & 11.18 & 10.82 & $8.54 \pm 0.06$ & $-0.037 \pm 0.018$ & 0.857 \\
\hline NGC 7819 & $\mathrm{Sc}$ & -20.68 & 10.39 & 8.36 & $8.49 \pm 0.06$ & $-0.12 \pm 0.02$ & 0.985 \\
\hline NGC 7824 & Sab & -21.47 & 11.30 & 11.60 & $8.48 \pm 0.05$ & $-0.03 \pm 0.05$ & 0.991 \\
\hline UGC 00005 & $\mathrm{Sbc}$ & -21.50 & 10.83 & 9.56 & $8.52 \pm 0.04$ & $-0.055 \pm 0.009$ & 0.987 \\
\hline UGC 00036 & SABab & -20.94 & 10.98 & 8.64 & $8.51 \pm 0.06$ & $-0.13 \pm 0.04$ & 0.997 \\
\hline UGC 01918 & $\mathrm{SBb}$ & -20.53 & 10.56 & 7.95 & $8.53 \pm 0.05$ & $-0.08 \pm 0.04$ & 0.996 \\
\hline UGC 02311 & SBbc & -21.59 & 10.74 & 7.95 & $8.52 \pm 0.04$ & $-0.050 \pm 0.010$ & 0.942 \\
\hline UGC 03253 & $\mathrm{SBb}$ & -20.43 & 10.68 & 6.54 & $8.52 \pm 0.04$ & $-0.125 \pm 0.012$ & 0.938 \\
\hline UGC 03973 & SBbc & -21.86 & 10.74 & 7.66 & $8.50 \pm 0.05$ & $0.008 \pm 0.009$ & 0.209 \\
\hline UGC 04195 & $\mathrm{SBb}$ & -20.68 & 10.50 & 8.02 & $8.52 \pm 0.07$ & $-0.020 \pm 0.013$ & 0.789 \\
\hline UGC 04262 & SABbc & -21.26 & 10.60 & 12.31 & $8.49 \pm 0.10$ & $-0.25 \pm 0.03$ & 0.953 \\
\hline UGC 04308 & $\mathrm{SBc}$ & -20.95 & 10.30 & 6.73 & $8.52 \pm 0.06$ & $-0.087 \pm 0.011$ & 0.964 \\
\hline UGC 04375 & Sbc & -19.90 & 10.16 & 6.12 & $8.49 \pm 0.04$ & $-0.121 \pm 0.015$ & 0.948 \\
\hline UGC 05108 & $\mathrm{SBb}$ & -21.51 & 10.89 & 17.80 & $8.49 \pm 0.04$ & $-0.12 \pm 0.05$ & 0.977 \\
\hline UGC 07012 & SABcd & -19.68 & 9.45 & 5.28 & $8.40 \pm 0.08$ & $-0.11 \pm 0.03$ & 0.995 \\
\hline UGC 08781 & $\mathrm{SBb}$ & -21.69 & 11.05 & 17.57 & $8.48 \pm 0.06$ & $-0.02 \pm 0.03$ & 0.904 \\
\hline UGC 09291 & Scd & -20.32 & 10.34 & 7.64 & $8.44 \pm 0.06$ & $-0.18 \pm 0.03$ & 0.986 \\
\hline UGC 09476 & $\mathrm{Sbc}$ & -20.49 & 10.21 & 5.89 & $8.49 \pm 0.05$ & $-0.057 \pm 0.012$ & 0.971 \\
\hline UGC 09777 & Sbc & -20.47 & 10.31 & 7.67 & $8.48 \pm 0.08$ & $-0.00 \pm 0.06$ & 0.266 \\
\hline
\end{tabular}


L. Sánchez-Menguiano et al.: Shape of the oxygen abundance profiles in CALIFA face-on spiral galaxies

Table A.1. continued.

\begin{tabular}{llcccccc}
\hline \hline Name & $\begin{array}{l}\text { Morph } \\
\text { type }\end{array}$ & $\begin{array}{c}M_{g} \\
{[\mathrm{mag}]}\end{array}$ & $\begin{array}{c}\log _{10} \text { Mass } \\
{\left[M_{\odot}\right]}\end{array}$ & $\begin{array}{c}r_{\mathrm{e}} \\
{[\mathrm{kpc}]}\end{array}$ & $\begin{array}{c}{[\mathrm{O} / \mathrm{H}]_{r_{\mathrm{e}}}} \\
{[\mathrm{dex}]}\end{array}$ & $\begin{array}{c}\alpha_{\mathrm{O} / \mathrm{H}} \\
{\left[\mathrm{dex} / r_{\mathrm{e}}\right]}\end{array}$ & c.c. \\
& (b) & (c) & $(\mathrm{d})$ & $(\mathrm{e})$ & $(\mathrm{f})$ & $(\mathrm{g})$ & $(\mathrm{h})$ \\
\hline UGC 09842 & SBbc & -21.18 & 10.63 & 14.27 & $8.50 \pm 0.06$ & $-0.031 \pm 0.015$ & 0.533 \\
UGC 11649 & SBab & -20.84 & 10.66 & 6.23 & $8.53 \pm 0.07$ & $-0.10 \pm 0.03$ & 0.890 \\
UGC 12224 & Sc & -20.48 & 9.97 & 7.82 & $8.47 \pm 0.06$ & $-0.13 \pm 0.05$ & 0.987 \\
UGC 12633 & SABab & -20.35 & 10.37 & 7.09 & $8.47 \pm 0.00$ & $-0.03 \pm 0.02$ & 0.666 \\
UGC 12816 & Sc & -20.35 & 9.82 & 8.56 & $8.42 \pm 0.06$ & $-0.07 \pm 0.02$ & 0.767 \\
UGC A021 & SBdm & -19.48 & 9.45 & 5.12 & $8.35 \pm 0.06$ & $-0.11 \pm 0.06$ & 0.700 \\
\hline
\end{tabular}

